\title{
Highly Selective C-H Functionalization/Halogenation of Acetanilide
}

Xiaobing Wan, ${ }^{\dagger}$ Zhongxun Ma, ${ }^{\mathbb{I}}$ Bijie Li, ${ }^{\dagger}$ Keya Zhang, ${ }^{\dagger}$ Shaokui Cao, ${ }^{\mathbb{I l}}$ Shiwei Zhang ${ }^{\dagger}$ and Zhangjie Shi ${ }^{\dagger}$

${ }^{\dagger}$ The Key Laboratory of Bioorganic Chemistry and Molecular Engineering of Ministry of Education, College of Chemistry, Peking University, Beijing 100871, China and State Key Laboratory of Organometallic Chemistry, Chinese Academy of Sciences, Shanghai 200032, China.

${ }^{I I S c h o o l ~ o f ~ M a t e r i a l ~ S c i e n c e ~ a n d ~ E n g i n e e r i n g, ~ Z h e n g z h o u ~ U n i v e r s i t y, ~ Z h e n g z h o u, ~}$ Henan 450052, China

\section{Table of contents}

\begin{tabular}{l|l}
\hline Table & Page \\
\hline General & S2 \\
\hline Analytical and spectral data for compounds $\mathbf{2 a - 2 s}$ & S3-S7 \\
\hline NMR Spectrum of compounds $\mathbf{2 a - 2 s}$ & S8-S25 \\
\hline X-ray data of compound $\mathbf{2 a}, \mathbf{2 n}$ & S26-S31 \\
\hline
\end{tabular}




\section{Experimental Section}

General. All the reactions were carried out under dry nitrogen atmosphere. DCE was freshly distilled over $\mathrm{P}_{2} \mathrm{O}_{5}$. Anhydrous $\mathrm{Cu}(\mathrm{OAc})_{2}$ and $\mathrm{CuCl}_{2}$ were purchased from Acros Chemical and used without further purification. $\mathrm{Pd}(\mathrm{OAc})_{2}$ was purchased from Strem Chemical. ${ }^{1} \mathrm{H}$ NMR (200 MHz) and ${ }^{13} \mathrm{C}$ NMR $(50 \mathrm{MHz})$ were registered on Varian $200 \mathrm{M}$ spectrometers with $\mathrm{CDCl}_{3}$ as solvent and tetramethylsilane (TMS) as internal standard. Chemical shifts were reported in units (ppm) by assigning TMS

resonance in the ${ }^{1} \mathrm{H}$ spectrum as $0.00 \mathrm{ppm}$ and $\mathrm{CDCl}_{3}$ resonance in the ${ }^{13} \mathrm{C}$ spectrum as $77.0 \mathrm{ppm}$. All coupling constants ( $J$ values) were reported in Hertz $(\mathrm{Hz})$. Column chromatography was performed on silica gel 200-300 mesh. IR, X-ray, GC, MS, and HRMS were performed by the State-authorized Analytical Center in Peking University.

\section{General procedures for halogenation of acetanilide:}

Acetanilide $1(0.5 \mathrm{mmol}), \mathrm{Pd}(\mathrm{OAc})_{2}(0.05 \mathrm{mmol})$, anhydrous $\mathrm{Cu}(\mathrm{OAc})_{2}(1.0 \mathrm{mmol})$ and anhydrous $\mathrm{CuCl}_{2}(1.0 \mathrm{mmol})$ were added to an oven-dried Schlenck tube. The septumsealed tube was evacuated and refilled with $\mathrm{N}_{2}$ three times. DCE (4.0 mL) was added via syringe. The reaction mixture was heated at $90{ }^{\circ} \mathrm{C}$ in an oil bath for $48 \mathrm{~h}$. After the reaction was complete, the product $\mathbf{2}$ was obtained by silica gel flash chromatography with petroleum ethers:EtOAc mixtures as eluent. 
N-(2-chlorophenyl)acetamide (2a). ${ }^{1}$ Starting from $0.0675 \mathrm{~g}$ 1a to afford 2a $0.0679 \mathrm{~g}$. yield $80 \%$, mp 90-91 ${ }^{\circ} \mathrm{C} .{ }^{1} \mathrm{H}$ NMR $\left(\mathrm{CDCl}_{3}, 200 \mathrm{MHz}\right): \delta 8.25(\mathrm{~d}, 1 \mathrm{H}, J=8.2 \mathrm{~Hz})$, 7.60 (bs, $1 \mathrm{H}), 7.28$ (d, $1 \mathrm{H}, J=7.8 \mathrm{~Hz}), 7.22-7.14$ (m, $1 \mathrm{H}), 6.99-6.92(\mathrm{~m}, 1 \mathrm{H}), 2.15$ (s, $3 \mathrm{H}) .{ }^{13} \mathrm{C} \mathrm{NMR}\left(\mathrm{CDCl}_{3}, 50 \mathrm{MHz}\right): \delta 168.4,134.3,128.7,127.2,124.6,123.1,122.1$, 24.3. MS $\left(\mathrm{C}_{8} \mathrm{H}_{8} \mathrm{ClNO}\right): 169\left(\mathrm{M}^{+},{ }^{35} \mathrm{Cl}\right), 171\left(\mathrm{M}^{+},{ }^{37} \mathrm{Cl}\right)$. IR $\left(\mathrm{KBr}, \mathrm{cm}^{-1}\right)$ : v 3244, 1661, 1531, 1439, 1302, 1060.

N-(2-chlorophenyl)benzamide (2c). ${ }^{2}$ Starting from $0.0985 \mathrm{~g} \mathbf{1 c}$ to afford $2 \mathrm{c} 0.0301 \mathrm{~g}$. yield $26 \%$, mp 104-105 ${ }^{\circ} \mathrm{C} .{ }^{1} \mathrm{H}$ NMR $\left(\mathrm{CDCl}_{3}, 200 \mathrm{MHz}\right): \delta 8.59-8.56(\mathrm{~m}, 1 \mathrm{H}), 8.46$ (bs, $1 \mathrm{H})$, 7.94-7.91 (m, $2 \mathrm{H})$, 7.61-7.26 (m, $5 \mathrm{H})$, 7.11-7.06 (m, $1 \mathrm{H}) .{ }^{13} \mathrm{C} \mathrm{NMR}\left(\mathrm{CDCl}_{3}\right.$, $50 \mathrm{MHz}): \delta$ 165.2, 134.7, 134.5, 132.2, 129.0, 128.9, 127.8, 127.1, 124.8, 123.1, 121.6. $\operatorname{MS}\left(\mathrm{C}_{13} \mathrm{H}_{10} \mathrm{ClNO}\right): 231\left(\mathrm{M}^{+},{ }^{35} \mathrm{Cl}\right), 233\left(\mathrm{M}^{+},{ }^{37} \mathrm{Cl}\right) . \mathrm{IR}\left(\mathrm{KBr}, \mathrm{cm}^{-1}\right):$ v 3285, 1652, 1531, 1440, 1311.

N-(2-chlorophenyl)pivalamide (2h). ${ }^{3}$ Starting from $0.0885 \mathrm{~g} \mathbf{1 h}$ to afford $\mathbf{2 h} 0.0708 \mathrm{~g}$. yield $67 \%$, mp $75-76{ }^{\circ} \mathrm{C} .{ }^{1} \mathrm{H}$ NMR $\left(\mathrm{CDCl}_{3}, 200 \mathrm{MHz}\right): \delta 8.43-8.39(\mathrm{~m}, 1 \mathrm{H}), 8.02(\mathrm{bs}$, $1 \mathrm{H}), 7.38-7.23$ (m, $2 \mathrm{H}), 7.06-6.98$ (m, $1 \mathrm{H}), 1.34$ (s, $9 \mathrm{H}) .{ }^{13} \mathrm{C} \mathrm{NMR}\left(\mathrm{CDCl}_{3}, 50 \mathrm{MHz}\right)$ : $\delta 176.6,134.7,128.8,127.7,124.3,122.8,121.3,40.1,27.5 . \mathrm{MS}\left(\mathrm{C}_{11} \mathrm{H}_{14} \mathrm{ClNO}\right): 211$ $\left(\mathrm{M}^{+},{ }^{35} \mathrm{Cl}\right), 213\left(\mathrm{M}^{+},{ }^{37} \mathrm{Cl}\right) . \mathrm{IR}\left(\mathrm{KBr}, \mathrm{cm}^{-1}\right):$ v 3435, 1692, 1593, 1513, 1436, 1305, 1156, 1053.

N-(2-chlorophenyl)-3-phenylpropanamide (2i). ${ }^{4}$ Starting from $0.1125 \mathrm{~g}$ 1i to afford 2i 0.0714g. yield $55 \%$, mp 118-119 ${ }^{\circ} \mathrm{C} .{ }^{1} \mathrm{H}$ NMR $\left(\mathrm{CDCl}_{3}, 200 \mathrm{MHz}\right): \delta 8.37$ (d, $1 \mathrm{H}, J$ $=8.0 \mathrm{~Hz}), 7.55(\mathrm{bs}, 1 \mathrm{H}), 7.36-7.18(\mathrm{~m}, 7 \mathrm{H}), 7.07-6.98(\mathrm{~m}, 1 \mathrm{H}), 3.08(\mathrm{t}, 2 \mathrm{H}, J=7.6$ $\mathrm{Hz}), 2.75(\mathrm{t}, 2 \mathrm{H}, J=7.6 \mathrm{~Hz}) .{ }^{13} \mathrm{C} \mathrm{NMR}\left(\mathrm{CDCl}_{3}, 50 \mathrm{MHz}\right): \delta 170.3,140.2,134.3,128.8$, 128.5, 128.2, 127.5, 126.2, 124.5, 122.7, 121.8, 39.2, 31.2. MS ( $\left.\mathrm{C}_{15} \mathrm{H}_{14} \mathrm{ClNO}\right): 259$ $\left(\mathrm{M}^{+},{ }^{35} \mathrm{Cl}\right), 261\left(\mathrm{M}^{+},{ }^{37} \mathrm{Cl}\right) . \mathrm{IR}\left(\mathrm{KBr}, \mathrm{cm}^{-1}\right): v 3278,1660,1529,1443,1301,1063$.

N-(2-chloro-4-methylphenyl)acetamide (2j). ${ }^{5}$ Starting from $0.0745 \mathrm{~g} \mathbf{1 j}$ to afford $\mathbf{2 j}$ 
0.0725g. yield $79 \%, \mathrm{mp} 115-116{ }^{\circ} \mathrm{C} .{ }^{1} \mathrm{H}$ NMR $\left(\mathrm{CDCl}_{3}, 200 \mathrm{MHz}\right): \delta 8.16(\mathrm{~d}, 1 \mathrm{H}, J=$ $8.2 \mathrm{~Hz}$ ), 7.61 (bs, $1 \mathrm{H}), 7.17$ (s, $1 \mathrm{H}), 7.05$ (d, $1 \mathrm{H}, J=8.2 \mathrm{~Hz}), 2.29$ (s, $3 \mathrm{H}), 2.21$ (s, 3 H). ${ }^{13} \mathrm{C} \mathrm{NMR}\left(\mathrm{CDCl}_{3}, 50 \mathrm{MHz}\right): \delta 168.3,134.7,132.0,129.2,128.2,122.6,121.8$, 24.6, 20.5. MS ( $\left.\mathrm{C}_{9} \mathrm{H}_{10} \mathrm{ClNO}\right): 183\left(\mathrm{M}^{+},{ }^{35} \mathrm{Cl}\right), 185\left(\mathrm{M}^{+},{ }^{37} \mathrm{Cl}\right) . \mathrm{IR}\left(\mathrm{KBr}, \mathrm{cm}^{-1}\right)$ : v 3279, $1661,1532,1297$

N-(2-chloro-5-methylphenyl)acetamide $\quad(\mathbf{2 k a}){ }^{1} \quad 5 \% \quad \mathrm{Pd}(\mathrm{OAc})_{2} . \quad$ Starting from 0.0745g 1k to afford 2 ka 0.0881g. yield $96 \%$, mp 91-92 ${ }^{\circ} \mathrm{C} .{ }^{1} \mathrm{H}$ NMR $\left(\mathrm{CDCl}_{3}, 200\right.$ MHz): $\delta 8.17$ (s, $1 \mathrm{H}), 7.56$ (bs, $1 \mathrm{H}), 7.21(\mathrm{~d}, 1 \mathrm{H}, J=8.2 \mathrm{~Hz}), 6.83(\mathrm{~d}, 1 \mathrm{H}, J=8.2 \mathrm{~Hz}$ ), 2.32 (s, $3 \mathrm{H}), 2.22$ (s, $3 \mathrm{H}) .{ }^{13} \mathrm{C} \mathrm{NMR}\left(\mathrm{CDCl}_{3}, 50 \mathrm{MHz}\right): \delta 168.3,137.7,134.1,128.4$, 125.4, 122.3, 119.7, 24.6, 21.1. MS ( $\left.\mathrm{C}_{9} \mathrm{H}_{10} \mathrm{ClNO}\right): 183\left(\mathrm{M}^{+},{ }^{35} \mathrm{Cl}\right), 185\left(\mathrm{M}^{+},{ }^{37} \mathrm{Cl}\right)$. IR $\left(\mathrm{KBr}, \mathrm{cm}^{-1}\right):$ v 3273, 1665, 1584, 1534, 1293, 1057.

N-(2-bromo-5-methylphenyl)acetamide (2kb). ${ }^{6}$ Starting from $0.0745 \mathrm{~g} \mathbf{1 k}$ to afford 2kb 0.0980g. yield $86 \%$, mp 120-121 ${ }^{\circ} \mathrm{C} .{ }^{1} \mathrm{H}$ NMR $\left(\mathrm{CDCl}_{3}, 200 \mathrm{MHz}\right)$ : $87.45-7.16$ (m, $4 \mathrm{H}), 2.35$ (s, $3 \mathrm{H}), 2.15$ (s, $3 \mathrm{H}) .{ }^{13} \mathrm{C} \mathrm{NMR}\left(\mathrm{CDCl}_{3}, 50 \mathrm{MHz}\right): \delta 169.2,138.3,137.1$, 132.3, 122.3, 119.4, 119.1, 24.3, 22.9. MS ( $\left.\mathrm{C}_{9} \mathrm{H}_{10} \mathrm{BrNO}\right): 227\left(\mathrm{M}^{+},{ }^{79} \mathrm{Br}\right), 229\left(\mathrm{M}^{+}\right.$, $\left.{ }^{81} \mathrm{Br}\right) . \mathrm{IR}\left(\mathrm{KBr}, \mathrm{cm}^{-1}\right)$ : v 3300, 1666, 1608, 1541, 1478, 1401, 1315, 1027.

N-(2-chloro-4-methoxyphenyl)acetamide (2l). ${ }^{7}$ Starting from $0.0825 \mathrm{~g} 1 \mathrm{ll}$ to afford $2 \mathrm{l}$ 0.0778g. yield $78 \%$, mp 114-115 ${ }^{\circ} \mathrm{C} .{ }^{1} \mathrm{H}$ NMR $\left(\mathrm{CDCl}_{3}, 200 \mathrm{MHz}\right): \delta 8.13(\mathrm{~d}, 1 \mathrm{H}, J=$ $9.0 \mathrm{~Hz}), 7.41$ (bs, $1 \mathrm{H}), 6.91(\mathrm{~d}, 1 \mathrm{H}, J=2.8 \mathrm{~Hz}), 6.81(\mathrm{dd}, 1 \mathrm{H}, J=9.0 \mathrm{~Hz}, J=2.8 \mathrm{~Hz})$, $3.77(\mathrm{~s}, 3 \mathrm{H}), 2.21(\mathrm{~s}, 3 \mathrm{H}) .{ }^{13} \mathrm{C} \mathrm{NMR}\left(\mathrm{CDCl}_{3}, 50 \mathrm{MHz}\right): \delta 168.1,156.2,127.8,124.1$, 123.4, 114.4, 113.1, 55.6, 24.5. MS $\left(\mathrm{C}_{9} \mathrm{H}_{10} \mathrm{ClNO}_{2}\right): 199\left(\mathrm{M}^{+},{ }^{35} \mathrm{Cl}\right), 201\left(\mathrm{M}^{+},{ }^{37} \mathrm{Cl}\right) . \mathrm{IR}$ $\left(\mathrm{KBr}, \mathrm{cm}^{-1}\right): \vee 3274,1657,1532,1284,1053$.

N-(2-chloro-5-methoxyphenyl)acetamide $\quad(\mathbf{2 m}){ }^{8} \quad 5 \% \quad \mathrm{Pd}(\mathrm{OAc})_{2}$. Starting from $0.0825 \mathrm{~g} \mathbf{1 m}$ to afford $\mathbf{2 m} 0.0780 \mathrm{~g}$. yield $78 \%$, mp 100-101 ${ }^{\circ} \mathrm{C} . \quad{ }^{1} \mathrm{H}$ NMR $\left(\mathrm{CDCl}_{3}\right.$, $200 \mathrm{MHz}$ ): $\delta 8.07$ (d, $1 \mathrm{H}, J=2.8 \mathrm{~Hz}), 7.61(\mathrm{bs}, 1 \mathrm{H}), 7.22$ (d, $1 \mathrm{H}, J=9.0 \mathrm{~Hz}), 6.51$ $(\mathrm{dd}, 1 \mathrm{H}, J=9.0 \mathrm{~Hz}, J=2.8 \mathrm{~Hz}), 3.79$ (s, $3 \mathrm{H}), 2.23$ (s, $3 \mathrm{H}) .{ }^{13} \mathrm{C} \mathrm{NMR}\left(\mathrm{CDCl}_{3}, 50\right.$ 
MHz): $\delta 168.4,158.8,135.2,129.1,113.6,110.8,106.6,55.4,24.8 . \mathrm{MS}\left(\mathrm{C}_{9} \mathrm{H}_{10} \mathrm{ClNO}_{2}\right)$ : $199\left(\mathrm{M}^{+},{ }^{35} \mathrm{Cl}\right), 201\left(\mathrm{M}^{+},{ }^{37} \mathrm{Cl}\right) . \mathrm{IR}\left(\mathrm{KBr}, \mathrm{cm}^{-1}\right):$ v 2923, 1678, 1593, 1528, 1463, 1308, 1260, 1028.

N-(2, 4-dichlorophenyl)acetamide (2n). ${ }^{9}$ Starting from $0.0848 \mathrm{~g}$ 1n to afford $2 \mathbf{n}$ 0.0275g. yield $27 \%$, mp 144-145 ${ }^{\circ} \mathrm{C} .{ }^{1} \mathrm{H} \mathrm{NMR}\left(\mathrm{CDCl}_{3}, 200 \mathrm{MHz}\right): \delta 8.34$ (d, $1 \mathrm{H}, J=$ $8.8 \mathrm{~Hz}), 7.56$ (bs, $1 \mathrm{H}), 7.38$ (d, $1 \mathrm{H}, J=2.4 \mathrm{~Hz}), 7.24(\mathrm{dd}, 1 \mathrm{H}, J=8.8 \mathrm{~Hz}, J=2.4 \mathrm{~Hz})$, 2.24 (s, $3 \mathrm{H}) .{ }^{13} \mathrm{C} \mathrm{NMR}\left(\mathrm{CDCl}_{3}, 50 \mathrm{MHz}\right): \delta 168.2,133.3,129.0,128.6,127.8,123.0$, 122.3, 24.8. MS $\left(\mathrm{C}_{8} \mathrm{H}_{7} \mathrm{Cl}_{2} \mathrm{NO}\right): 203\left(\mathrm{M}^{+},{ }^{35} \mathrm{Cl}\right), 205\left(\mathrm{M}^{+},{ }^{37} \mathrm{Cl}\right) . \mathrm{IR}\left(\mathrm{KBr}, \mathrm{cm}^{-1}\right)$ : v 3278, $1664,1525,1302,1059$.

N-(2-chloro-5-methoxy-4-methylphenyl)acetamide (20a). 5\% $\mathrm{Pd}(\mathrm{OAc})_{2}$. Starting from 0.0895g 10 to afford 2oa 0.0993g. yield $93 \%$, mp 119-120 ${ }^{\circ} \mathrm{C} .{ }^{1} \mathrm{H} \mathrm{NMR}\left(\mathrm{CDCl}_{3}\right.$, $200 \mathrm{MHz}$ ): $\delta 7.99$ (s, $1 \mathrm{H}), 7.55$ (bs, $1 \mathrm{H}), 7.08$ (s, $1 \mathrm{H}), 3.82$ (s, $3 \mathrm{H}), 2.22$ (s, $3 \mathrm{H}), 2.14$ (s, $3 \mathrm{H}) .{ }^{13} \mathrm{C} \mathrm{NMR}\left(\mathrm{CDCl}_{3}, 50 \mathrm{MHz}\right): \delta 168.4,156.6,132.9,129.7,123.3,112.8,103.8$, 55.5, 24.7, 15.4. MS $\left(\mathrm{C}_{10} \mathrm{H}_{12} \mathrm{ClNO}_{2}\right): 213\left(\mathrm{M}^{+},{ }^{35} \mathrm{Cl}\right), 215\left(\mathrm{M}^{+},{ }^{37} \mathrm{Cl}\right)$. HRMS: Anal. Calcd. for $\mathrm{C}_{10} \mathrm{H}_{12}{ }^{35} \mathrm{ClNO}_{2}$ 213.05566. Found: 213.05526. EA (\%): Anal. Calcd. for $\mathrm{C}_{10} \mathrm{H}_{12} \mathrm{ClNO}_{2} \mathrm{C}, 56.21 ; \mathrm{H}, 5.66 ; \mathrm{N}, 6.56$. Found: C, 56.10; H, 5.76; N, 6.41. IR (KBr, $\left.\mathrm{cm}^{-1}\right): v 3275,1667,1592,1523,1395,1291,1168,1019$.

N-(2-bromo-5-methoxy-4-methylphenyl)acetamide (2ob). Starting from 0.0895g 10 to afford 2ob $01161 \mathrm{~g}$. yield $90 \%$, mp 115-116 ${ }^{\circ} \mathrm{C} .{ }^{1} \mathrm{H}$ NMR $\left(\mathrm{CDCl}_{3}, 200 \mathrm{MHz}\right): \delta 7.91$ (s, $1 \mathrm{H}), 7.57$ (bs, $1 \mathrm{H}), 7.20$ (s, $1 \mathrm{H}), 3.79$ (s, $3 \mathrm{H}), 2.20$ (s, $3 \mathrm{H}), 2.11(\mathrm{~s}, 3 \mathrm{H}) .{ }^{13} \mathrm{C}$ NMR ( $\left.\mathrm{CDCl}_{3}, 50 \mathrm{MHz}\right): \delta 168.2,157.1,134.0,132.6,123.9,104.1,102.5,55.4,24.8$, 15.5. MS $\left(\mathrm{C}_{10} \mathrm{H}_{12} \mathrm{BrNO}_{2}\right)$ : $257\left(\mathrm{M}^{+},{ }^{79} \mathrm{Br}\right), 259\left(\mathrm{M}^{+},{ }^{81} \mathrm{Br}\right)$. HRMS: Anal. Calcd. for $\mathrm{C}_{10} \mathrm{H}_{12} \mathrm{BrNO}_{2}$ 257.00514 Found. 257.00560. EA (\%): Anal. Calcd. for $\mathrm{C}_{10} \mathrm{H}_{12} \mathrm{BrNO}_{2} \mathrm{C}$, 46.53; H, 4.69; N, 5.43. Found: C, 46.55; H, 4.65; N, 5.26. IR (KBr, $\left.\mathrm{cm}^{-1}\right): v 3274$, $1670,1519,1392,1166$ 
N-(2-chloro-3,4,5-trimethoxyphenyl)acetamide (2pa). Starting from $0.1125 \mathrm{~g}$ 1p to afford 2pa 0.0856g. yield $66 \%$, mp 104-105 ${ }^{\circ} \mathrm{C} .{ }^{1} \mathrm{H} \mathrm{NMR}\left(\mathrm{CDCl}_{3}, 200 \mathrm{MHz}\right): \delta 7.90$ (s, $1 \mathrm{H}), 7.55$ (bs, $1 \mathrm{H}), 3.91(\mathrm{~s}, 3 \mathrm{H}), 3.87(\mathrm{~s}, 3 \mathrm{H}), 3.85(\mathrm{~s}, 3 \mathrm{H}), 2.23(\mathrm{~s}, 3 \mathrm{H}) .{ }^{13} \mathrm{C}$ NMR ( $\left.\mathrm{CDCl}_{3}, 50 \mathrm{MHz}\right): \delta 168.4,152.1,149.5,139.2,130.8,108.6,100.9,61.1,55.9$, 24.6. MS $\left(\mathrm{C}_{11} \mathrm{H}_{14} \mathrm{ClNO}_{4}\right)$ : $259\left(\mathrm{M}^{+},{ }^{35} \mathrm{Cl}\right), 261\left(\mathrm{M}^{+},{ }^{37} \mathrm{Cl}\right)$. HRMS: Anal. Calcd. for $\mathrm{C}_{11} \mathrm{H}_{14}{ }^{35} \mathrm{ClNO}_{4}$ 259.06114 Found. 259.06070. EA (\%):Anal. Calcd. for $\mathrm{C}_{11} \mathrm{H}_{14} \mathrm{ClNO}_{4}$ C, 50.88; H, 5.43; N, 5.39. Found: C, 50.90; H, 5.29; N, 5.26. IR $\left(\mathrm{KBr}, \mathrm{cm}^{-1}\right): v 3281$, $1671,1590,1520,1398,1238,1112,1016$

N-(2-bromo-3,4,5-trimethoxyphenyl)acetamide (2pb). ${ }^{10}$ Starting from $0.1125 \mathrm{~g}$ 1p to afford 2pb 0.1383g. yield $91 \%$, mp 92-93 ${ }^{\circ} \mathrm{C} .{ }^{1} \mathrm{H}$ NMR $\left(\mathrm{CDCl}_{3}, 200 \mathrm{MHz}\right): \delta 7.86$ (s, $1 \mathrm{H}), 7.60$ (bs, $1 \mathrm{H}), 3.87$ (s, $3 \mathrm{H}), 3.84(\mathrm{~s}, 3 \mathrm{H}), 3.82(\mathrm{~s}, 3 \mathrm{H}), 2.20(\mathrm{~s}, 3 \mathrm{H}) .{ }^{13} \mathrm{C}$ NMR ( $\left.\mathrm{CDCl}_{3}, 50 \mathrm{MHz}\right): \delta 168.3,152.7,150.3,139.2,131.8,101.4$, 99.7, 61.0, 60.9, 55.9, 24.7. MS $\left(\mathrm{C}_{11} \mathrm{H}_{14} \mathrm{BrNO}_{4}\right): 303\left(\mathrm{M}^{+},{ }^{79} \mathrm{Br}\right), 305\left(\mathrm{M}^{+},{ }^{81} \mathrm{Br}\right) . \mathrm{IR}\left(\mathrm{KBr}, \mathrm{cm}^{-1}\right): v 3271$, $1669,1584,1393,1108$

Methyl 4-acetamido-5-chloro-2-methoxybenzoate (2qa). ${ }^{11}$ Starting from $0.1115 \mathrm{~g} 1 \mathrm{q}$ to afford 2qa 0.0386g. yield $30 \%, \mathrm{mp} 152-153{ }^{\circ} \mathrm{C} .{ }^{1} \mathrm{H} \mathrm{NMR}\left(\mathrm{CDCl}_{3}, 200 \mathrm{MHz}\right): \delta$ $8.23(\mathrm{~s}, 1 \mathrm{H}), 7.80(\mathrm{~s}, 2 \mathrm{H}), 3.86(\mathrm{~s}, 3 \mathrm{H}), 3.82(\mathrm{~s}, 3 \mathrm{H}), 2.23$ (s, $3 \mathrm{H}) .{ }^{13} \mathrm{C} \mathrm{NMR}\left(\mathrm{CDCl}_{3}\right.$, $50 \mathrm{MHz}): \delta 168.6,164.7,159.0,138.9,131.8,114.7,112.3,104.1,56.2,51.9,25.0 . \mathrm{MS}$ $\left(\mathrm{C}_{11} \mathrm{H}_{12} \mathrm{ClNO}_{4}\right): 257\left(\mathrm{M}^{+},{ }^{35} \mathrm{Cl}\right), 259\left(\mathrm{M}^{+},{ }^{37} \mathrm{Cl}\right) . \mathrm{IR}\left(\mathrm{KBr}, \mathrm{cm}^{-1}\right):$ v 3416, 1726, 1703, 1580, 1400, 1233, 1100.

Methyl 4-acetamido-5-bromo-2-methoxybenzoate (2qb). ${ }^{12}$ Starting from $0.1115 \mathrm{~g}$ $1 q$ to afford 2qb $0.1314 \mathrm{~g}$. yield $87 \%, \mathrm{mp} 170-172{ }^{\circ} \mathrm{C} .{ }^{1} \mathrm{H}$ NMR $\left(\mathrm{CDCl}_{3}, 200 \mathrm{MHz}\right): \delta$ 8.29 (s, $1 \mathrm{H}), 8.01(\mathrm{~s}, 1 \mathrm{H}), 7.75$ (bs, $1 \mathrm{H}), 3.91(\mathrm{~s}, 3 \mathrm{H}), 3.86(\mathrm{~s}, 3 \mathrm{H}), 2.26(\mathrm{~s}, 3 \mathrm{H}) .{ }^{13} \mathrm{C}$ $\mathrm{NMR}\left(\mathrm{CDCl}_{3}, 50 \mathrm{MHz}\right): \delta 168.6,164.6,159.6,140.0,134.9,115.3,104.2,101.7,56.1$, 51.9, 25.0. MS $\left(\mathrm{C}_{11} \mathrm{H}_{12} \mathrm{BrNO}_{4}\right): 301\left(\mathrm{M}^{+},{ }^{79} \mathrm{Br}\right), 303\left(\mathrm{M}^{+},{ }^{81} \mathrm{Br}\right) . \mathrm{IR}\left(\mathrm{KBr}, \mathrm{cm}^{-1}\right): v 3400$, 1685, 1457, 1404, 1237. 
1-(8-chloro-3,4-dihydroquinolin-1(2H)-yl)ethanone (2r). ${ }^{13} 5 \% \mathrm{Pd}(\mathrm{OAc})_{2}$. Starting from $0.0875 \mathrm{~g}$ 1r to afford 2 r $0.0995 \mathrm{~g}$. yield $95 \%$, mp 56-57 ${ }^{\circ} \mathrm{C} .{ }^{1} \mathrm{H} \mathrm{NMR}\left(\mathrm{CDCl}_{3}, 200\right.$ MHz): $\delta$ 7.30-7.12 (m, 3 H), 4.78-4.64 (m, 1 H), 2.95-2.65 (m, 2 H), 2.56-2.26 (m, 2 $\mathrm{H}), 2.03(\mathrm{~s}, 3 \mathrm{H}), 1.68-1.63(\mathrm{~m}, 1 \mathrm{H}) .{ }^{13} \mathrm{C} \mathrm{NMR}\left(\mathrm{CDCl}_{3}, 50 \mathrm{MHz}\right): \delta 170.9,139.0$, 137.8, 129.9, 128.0, 127.2, 126.2, 41.2, 26.6, 23.9, 21.6. MS ( $\left.\mathrm{C}_{11} \mathrm{H}_{12} \mathrm{ClNO}\right): 209\left(\mathrm{M}^{+}\right.$, $\left.{ }^{35} \mathrm{Cl}\right), 211\left(\mathrm{M}^{+},{ }^{37} \mathrm{Cl}\right) . \mathrm{IR}\left(\mathrm{KBr}, \mathrm{cm}^{-1}\right): v 1672,1464,1375,1267$. 


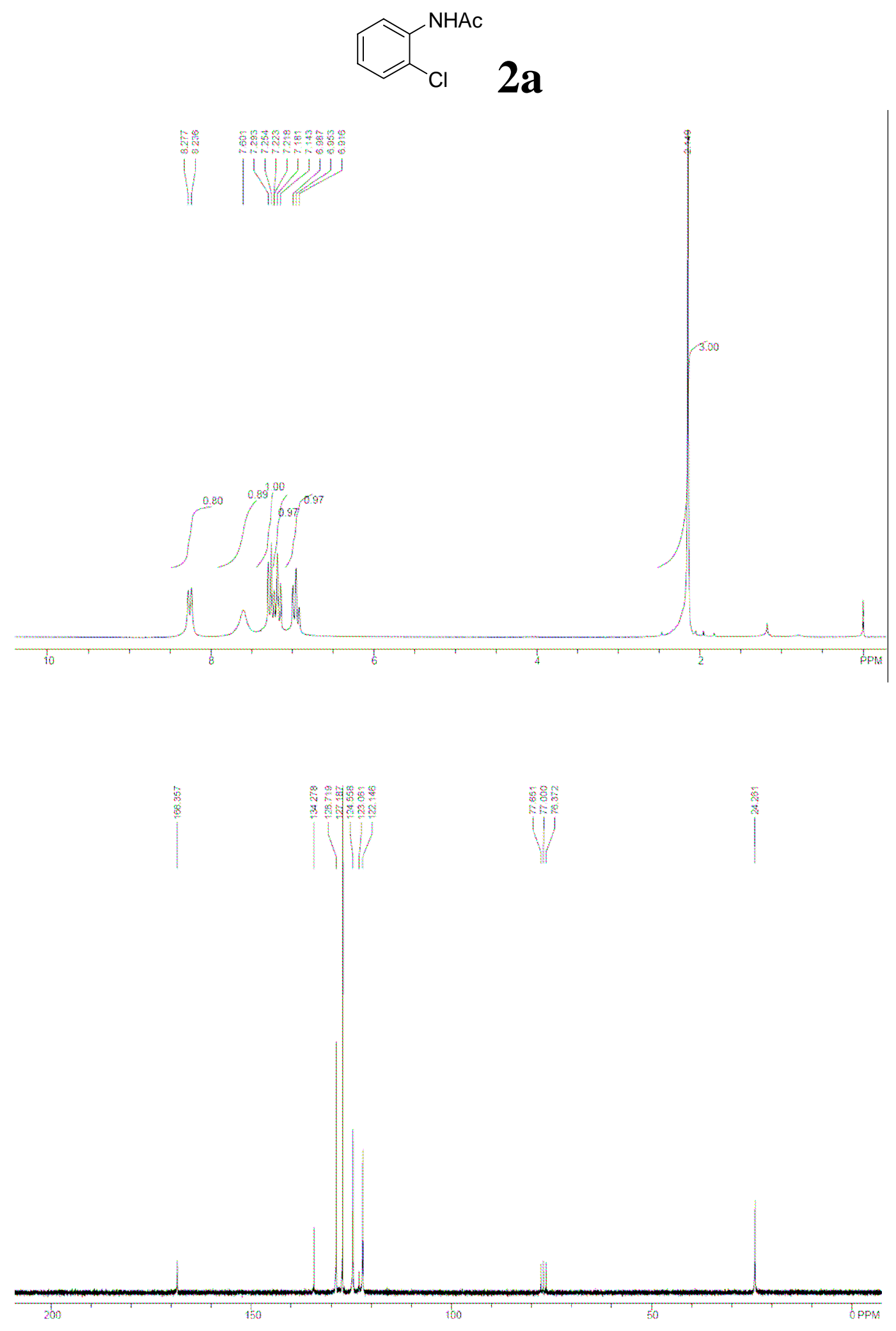



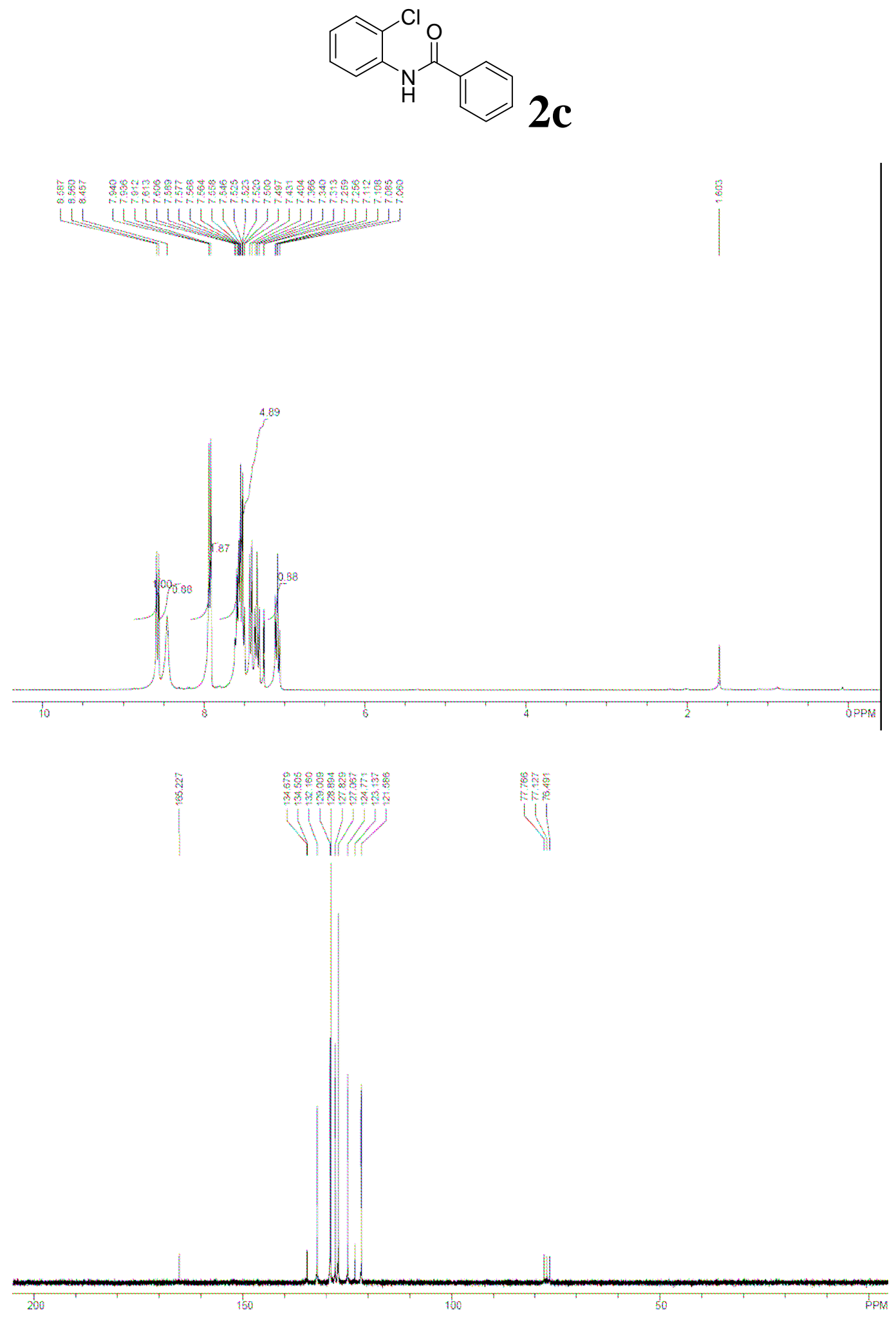

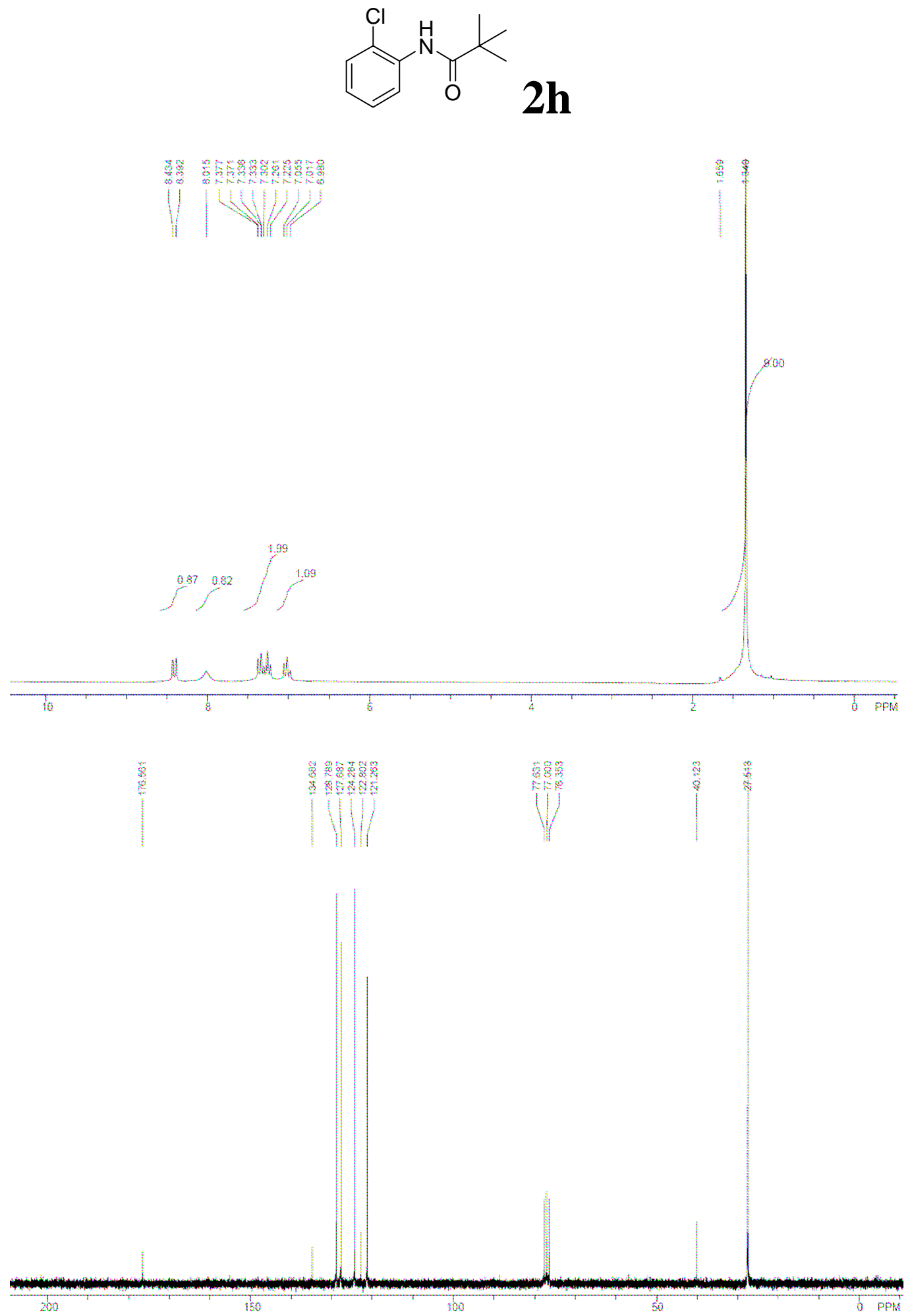


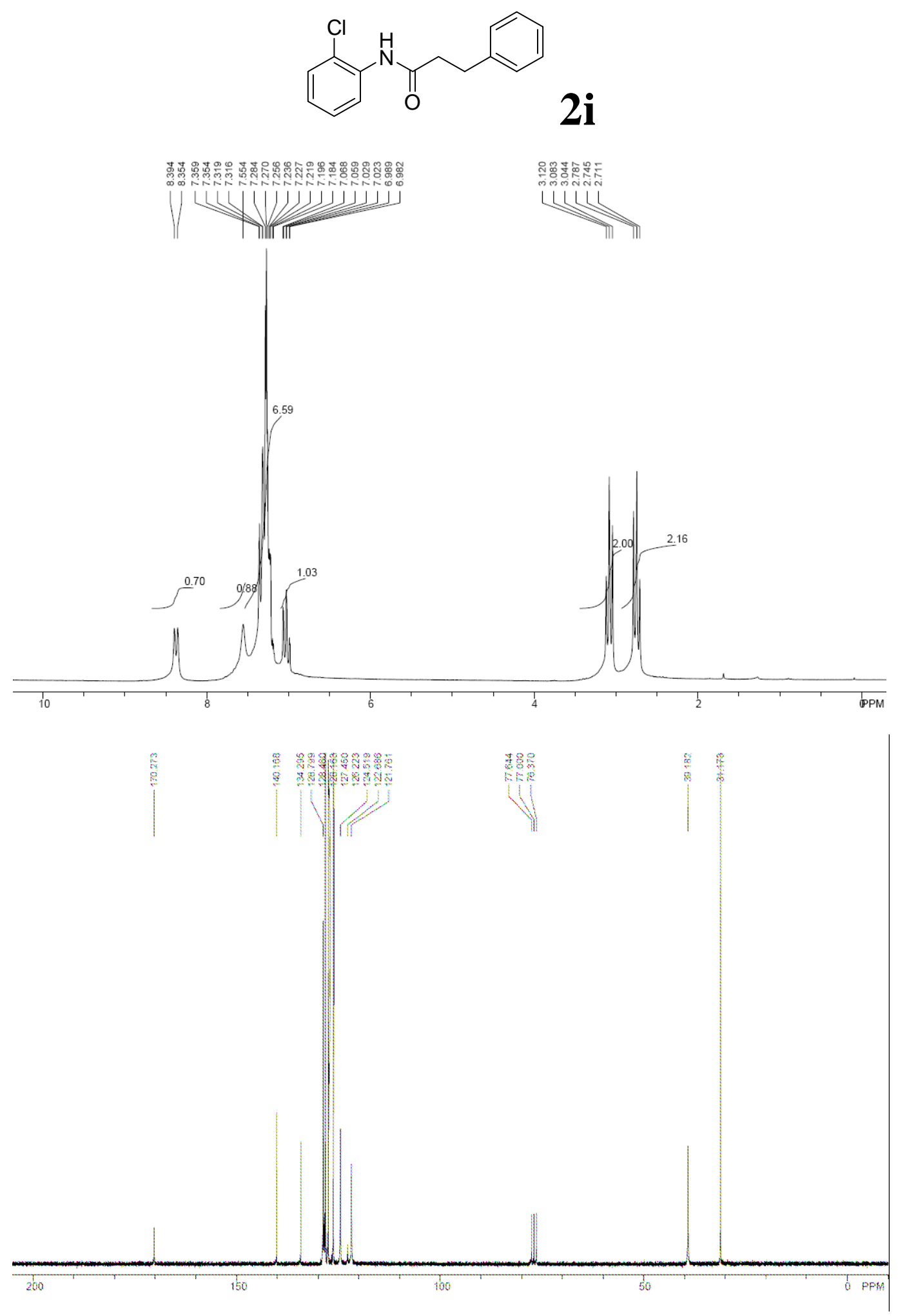



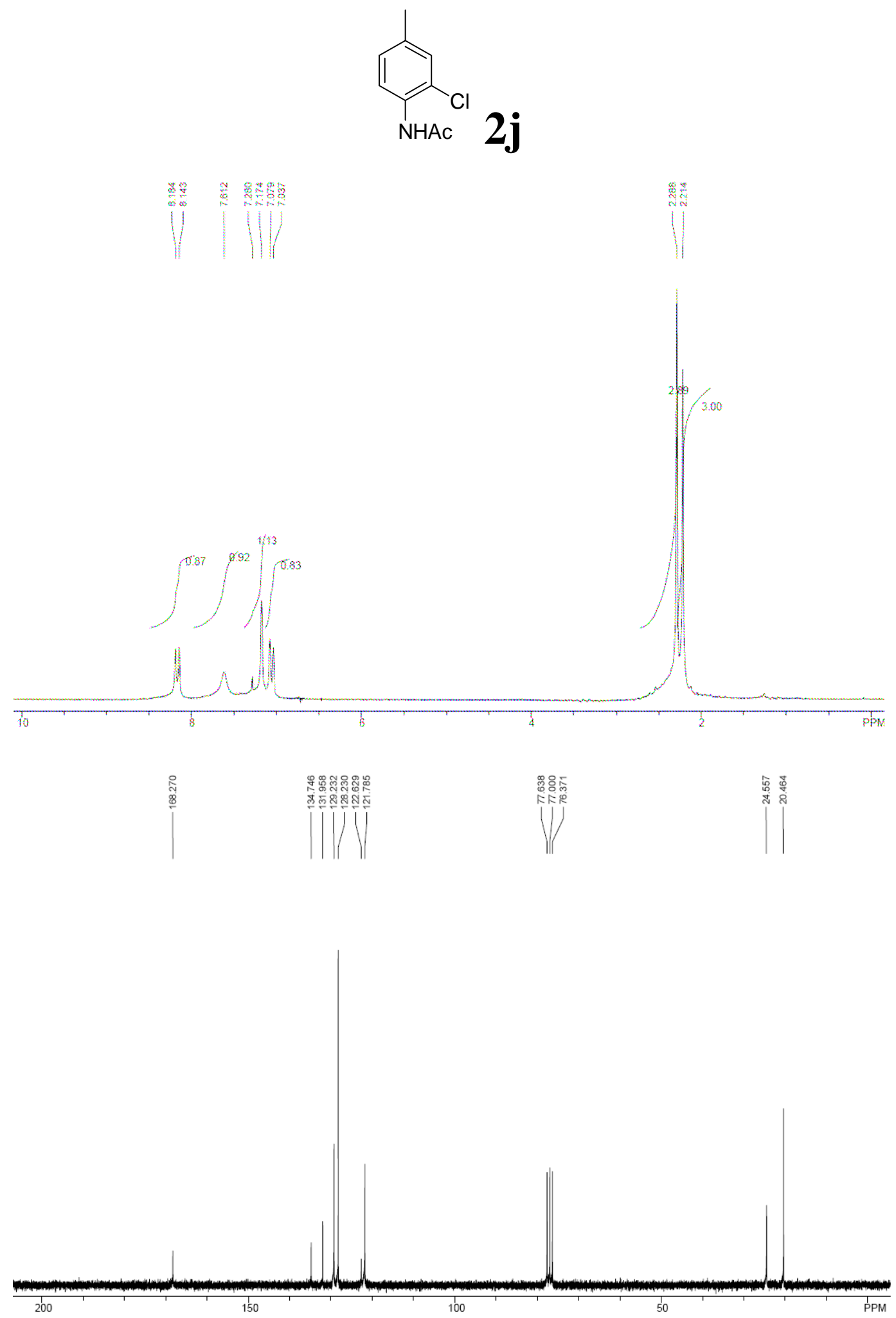

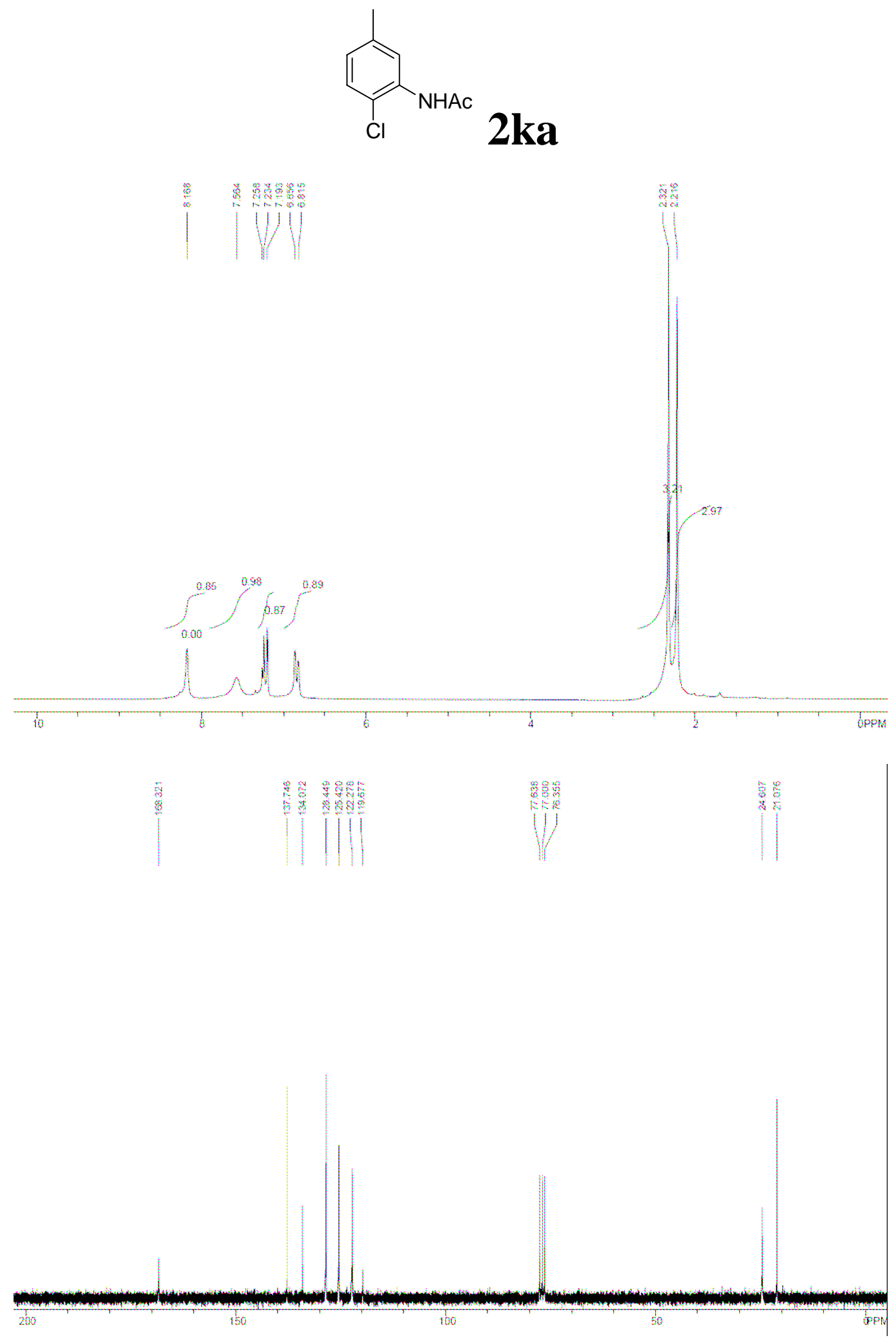

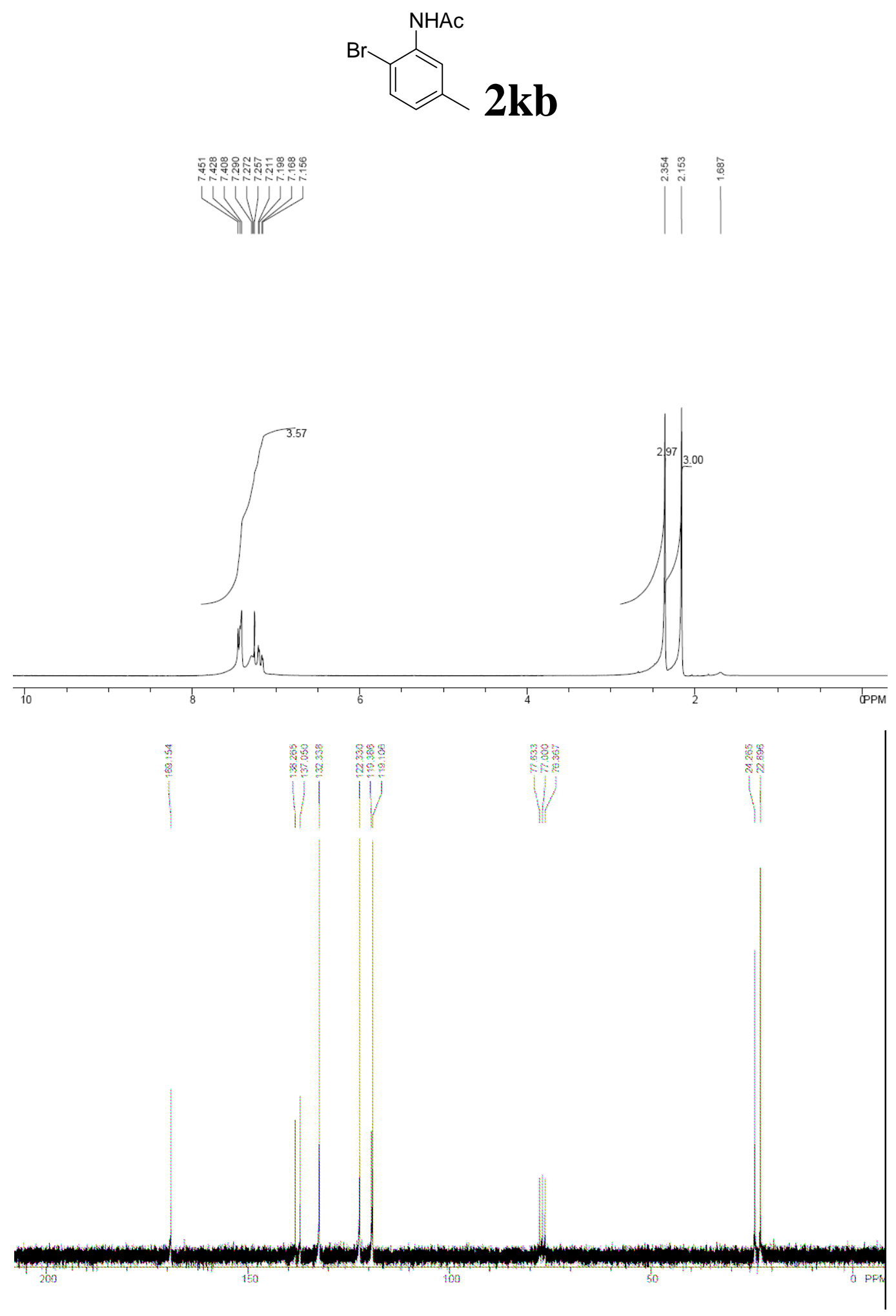
NHAc
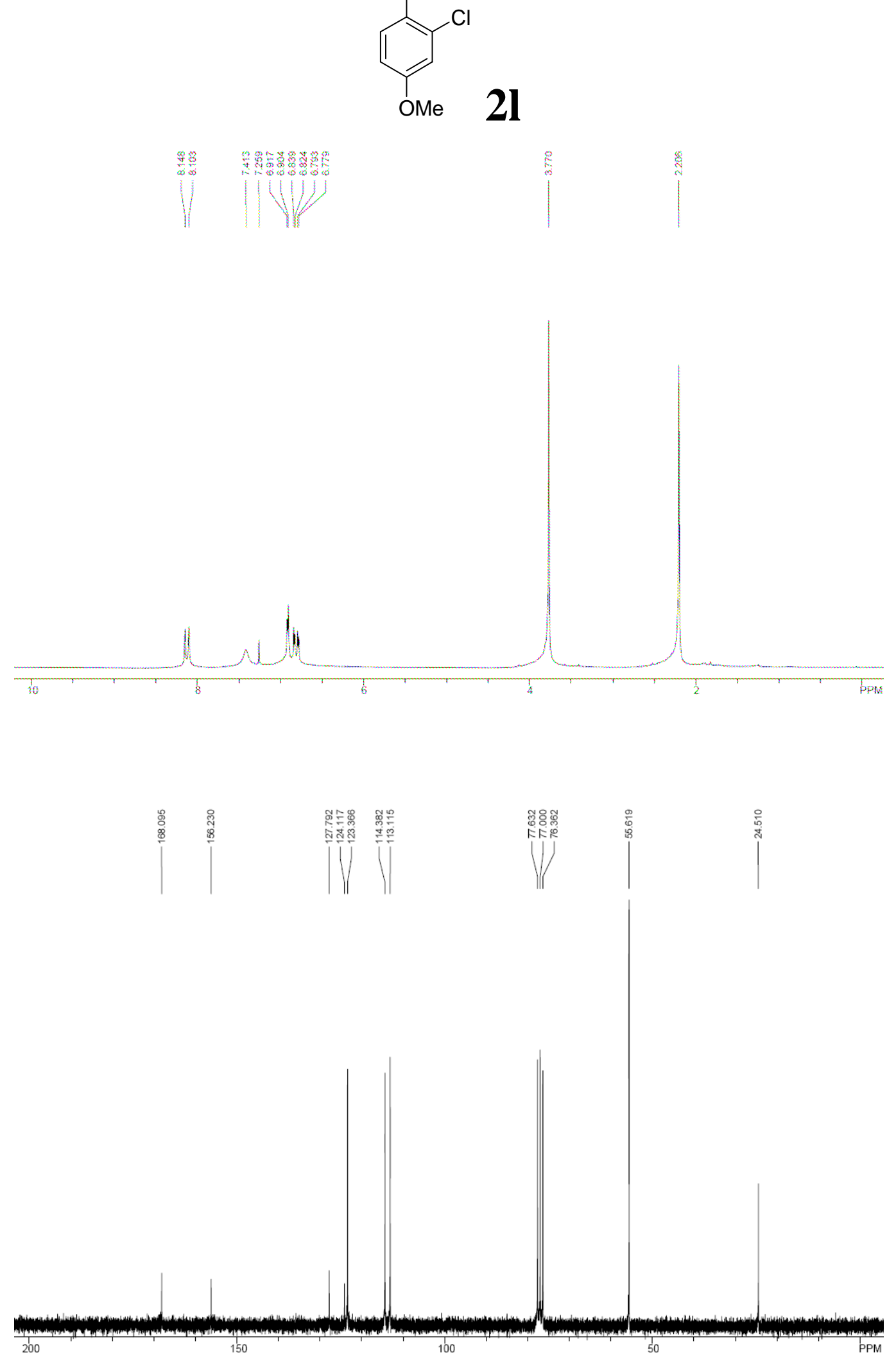

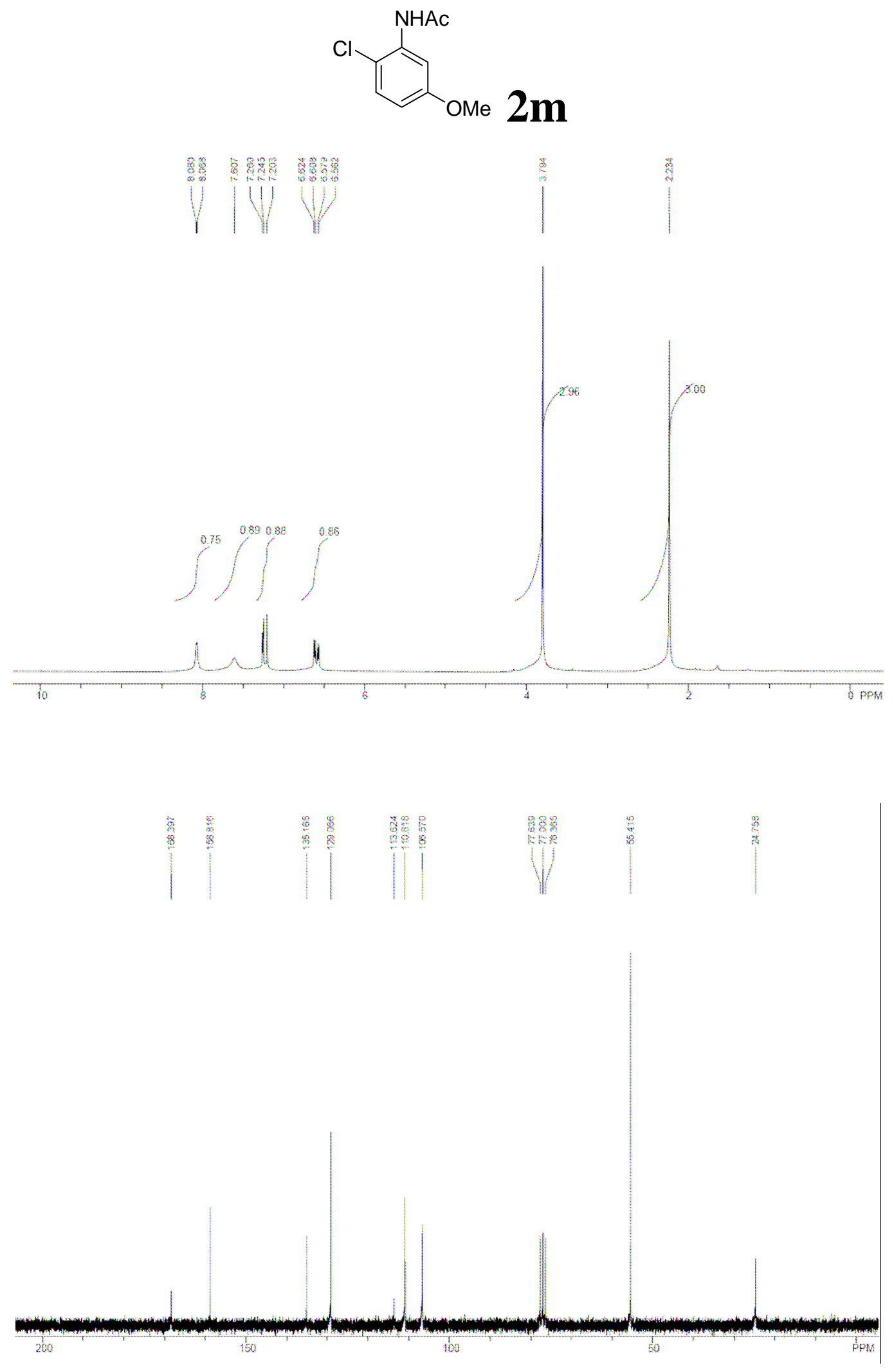

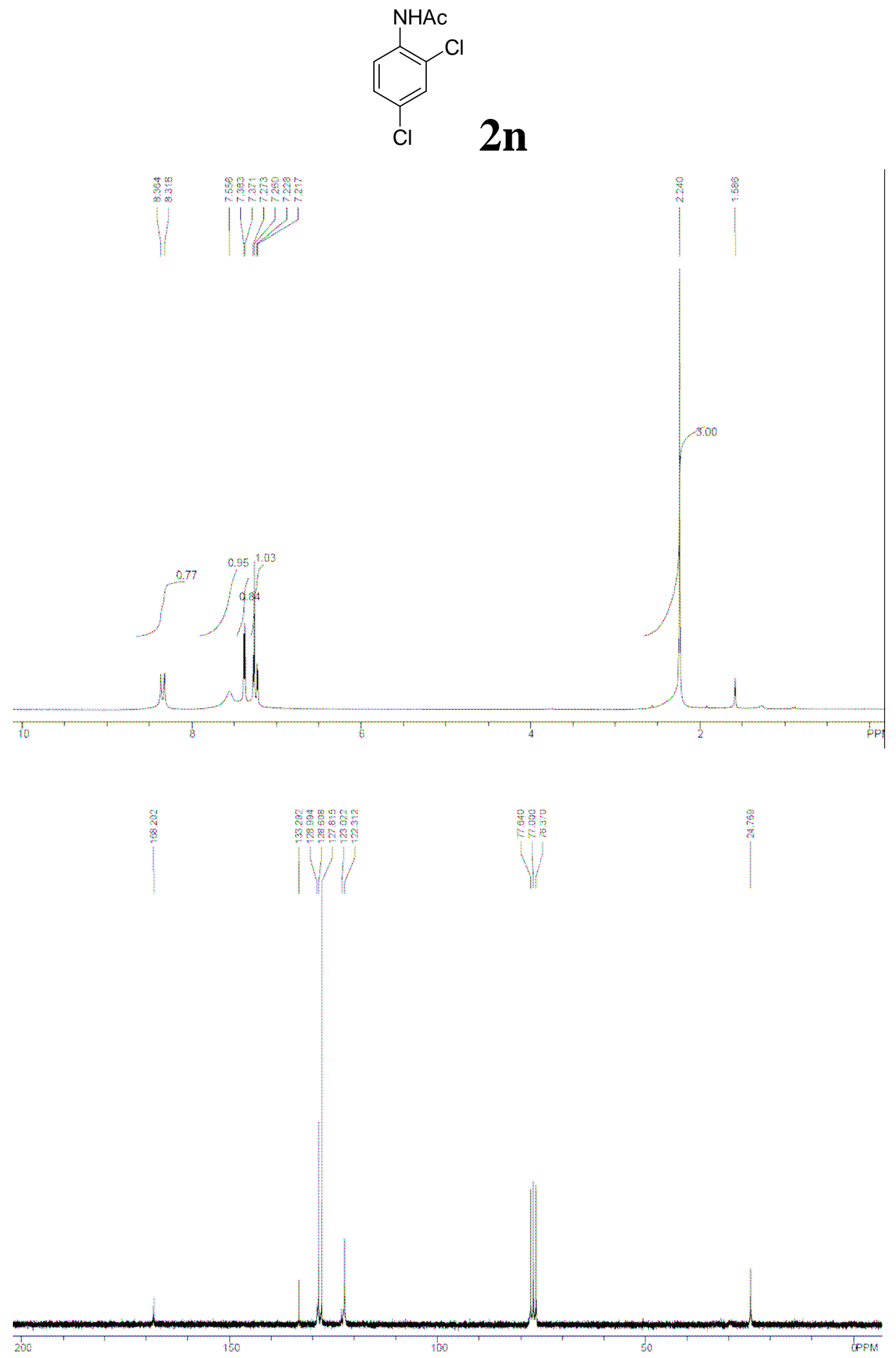

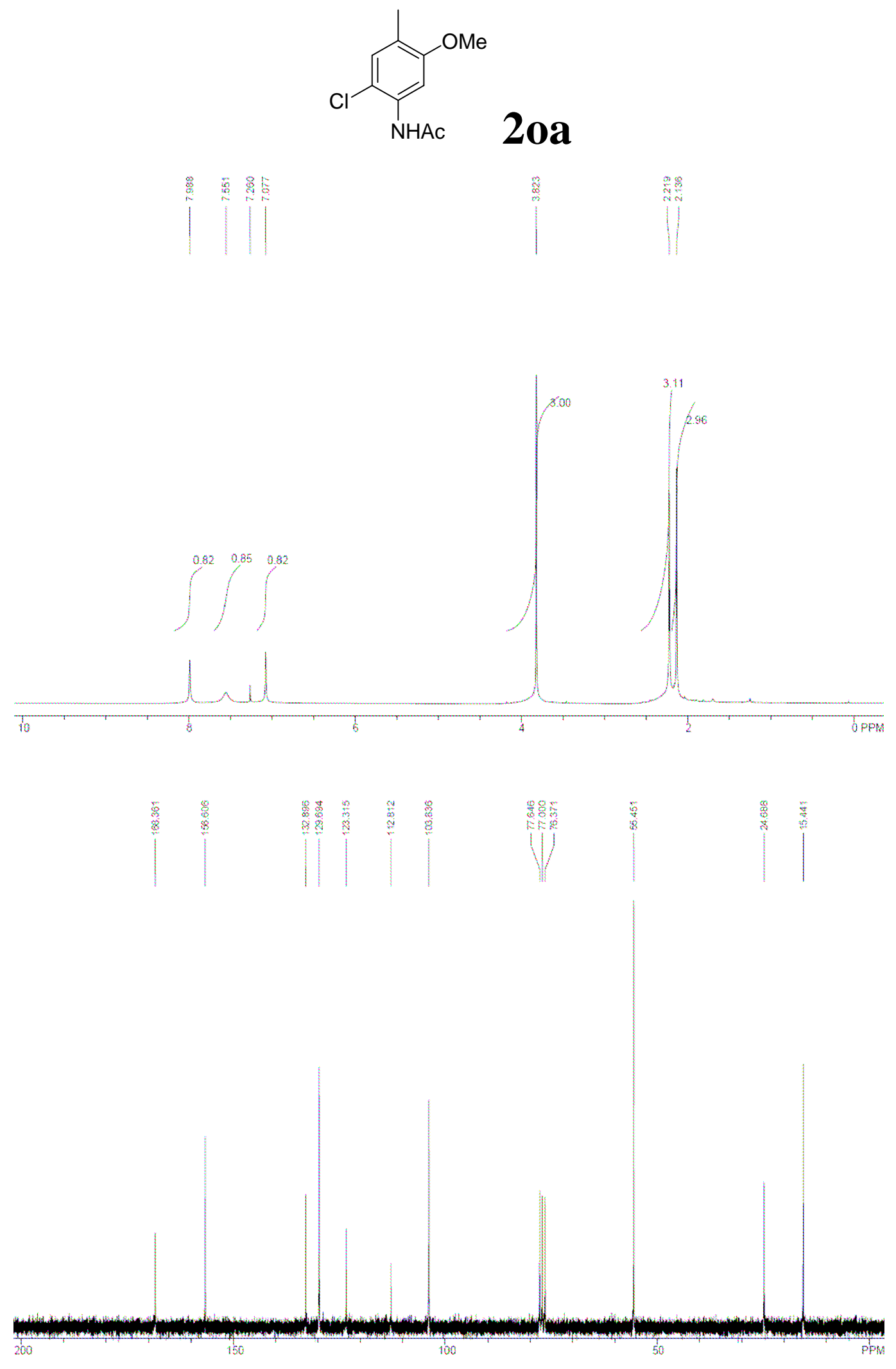

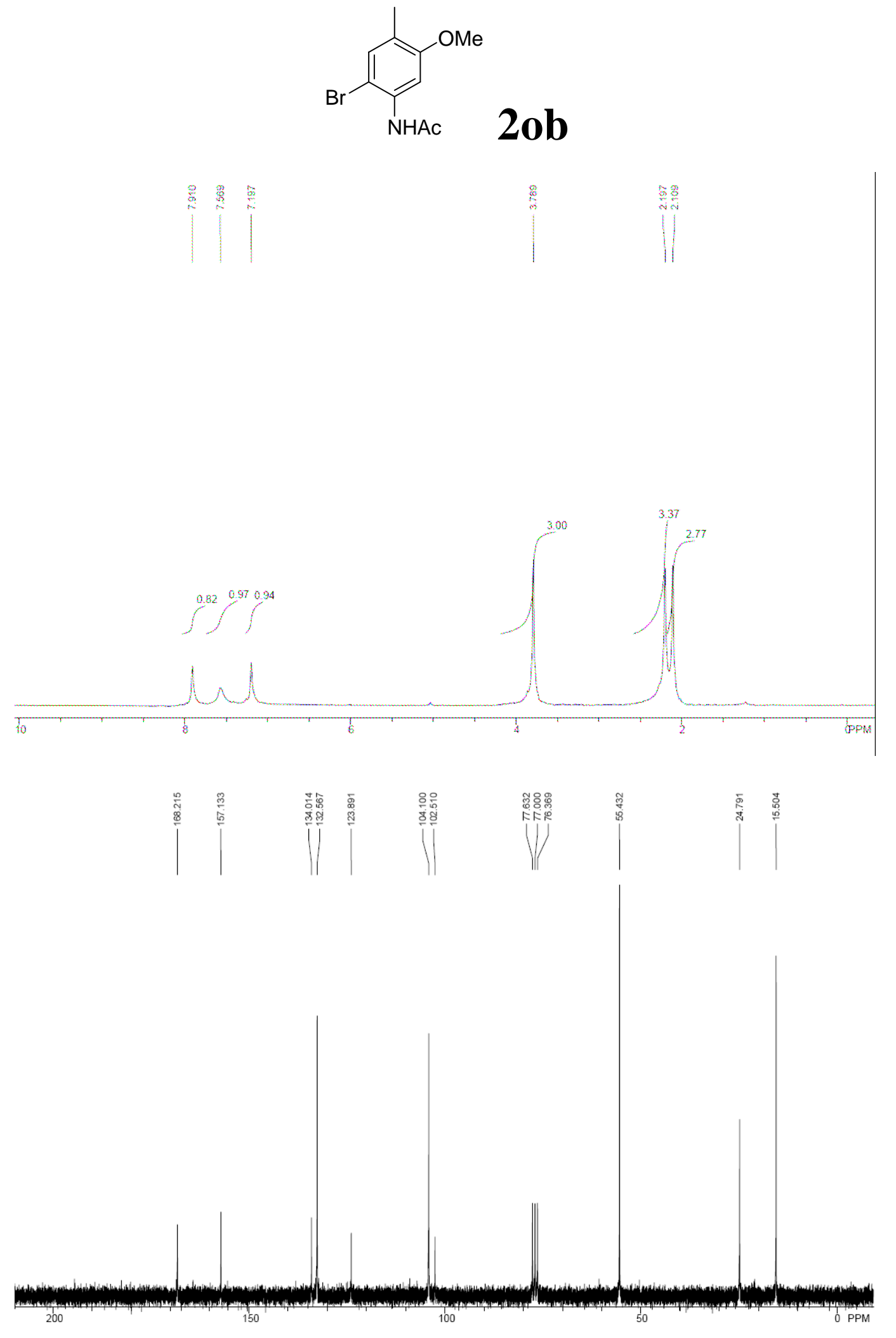

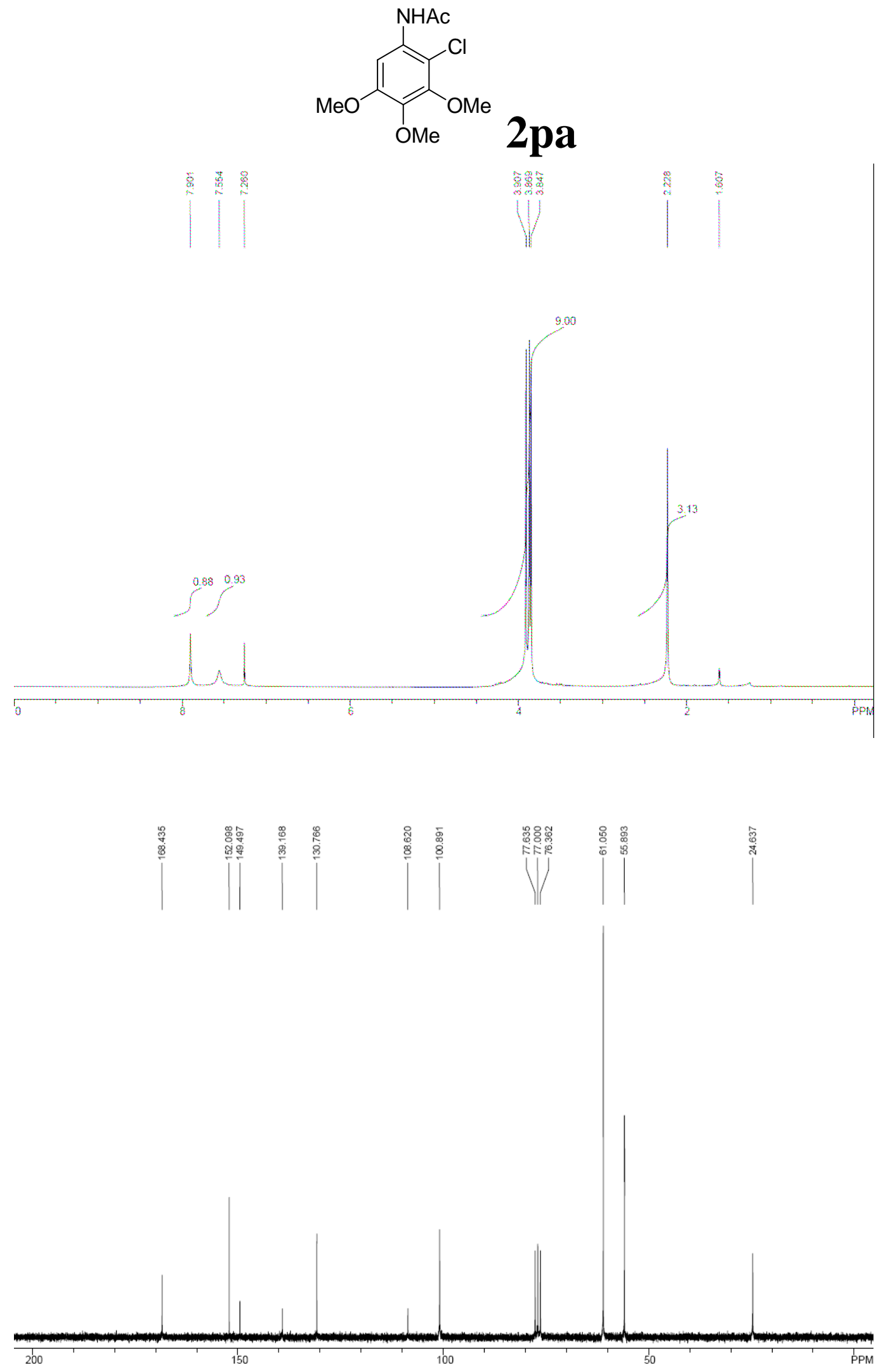

S20 

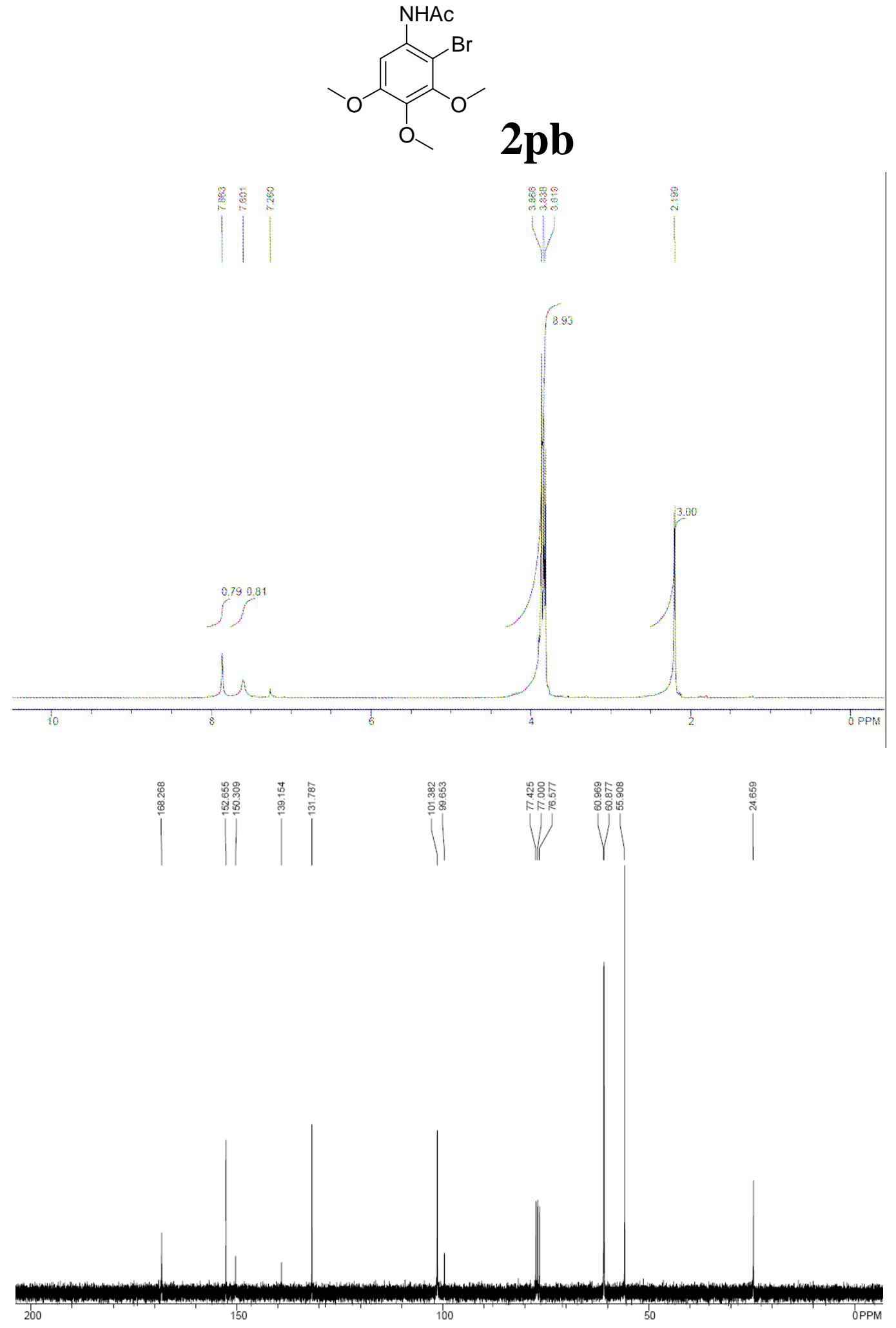


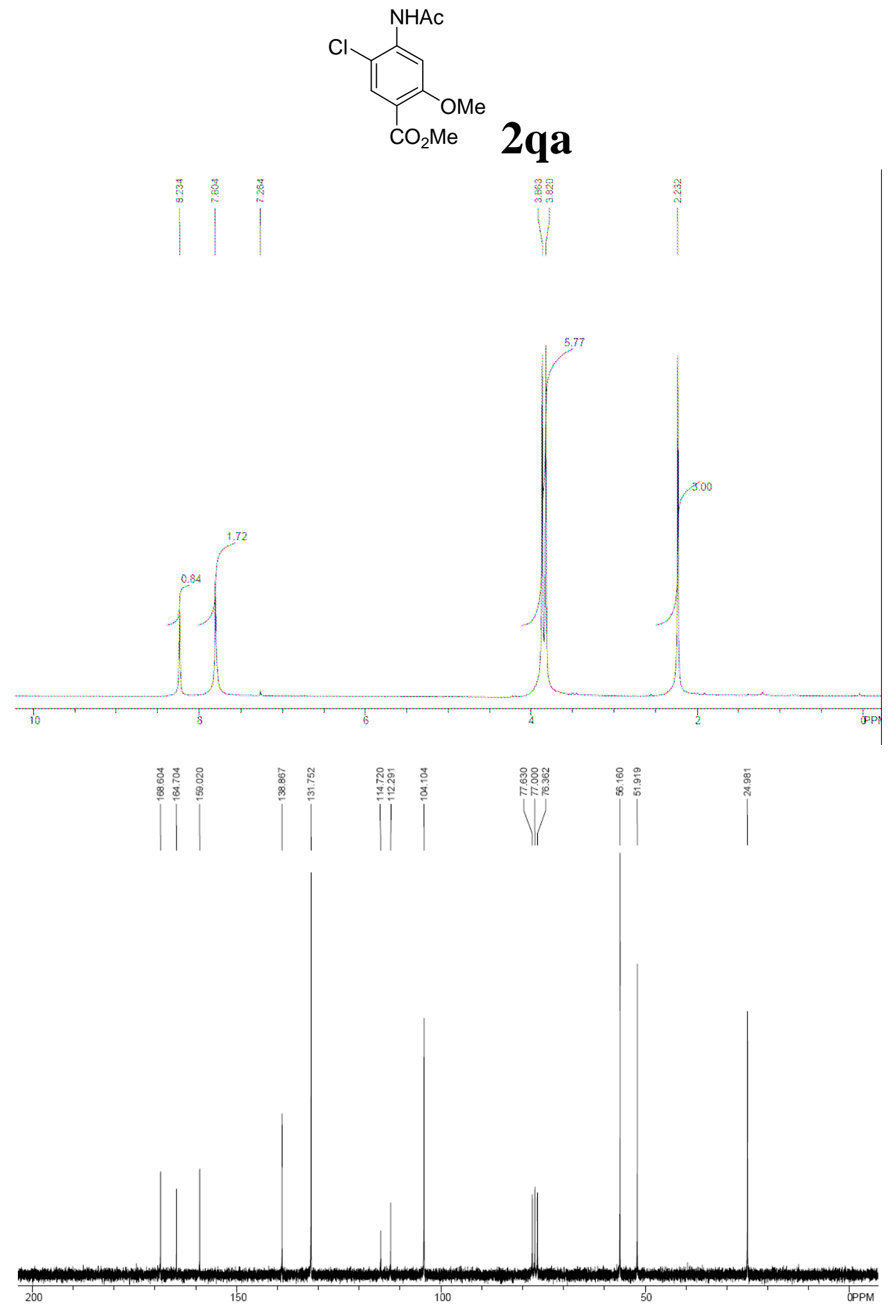




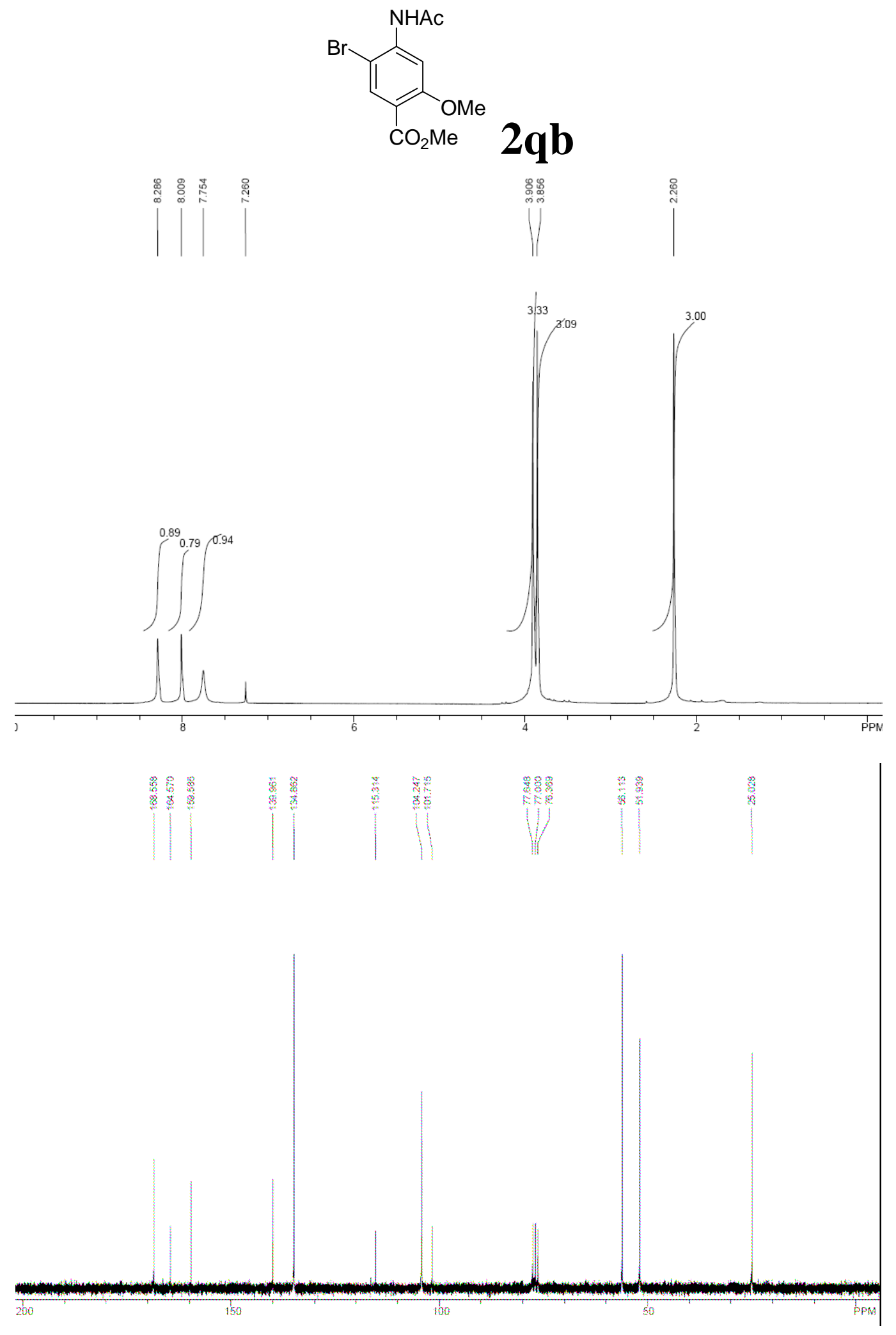



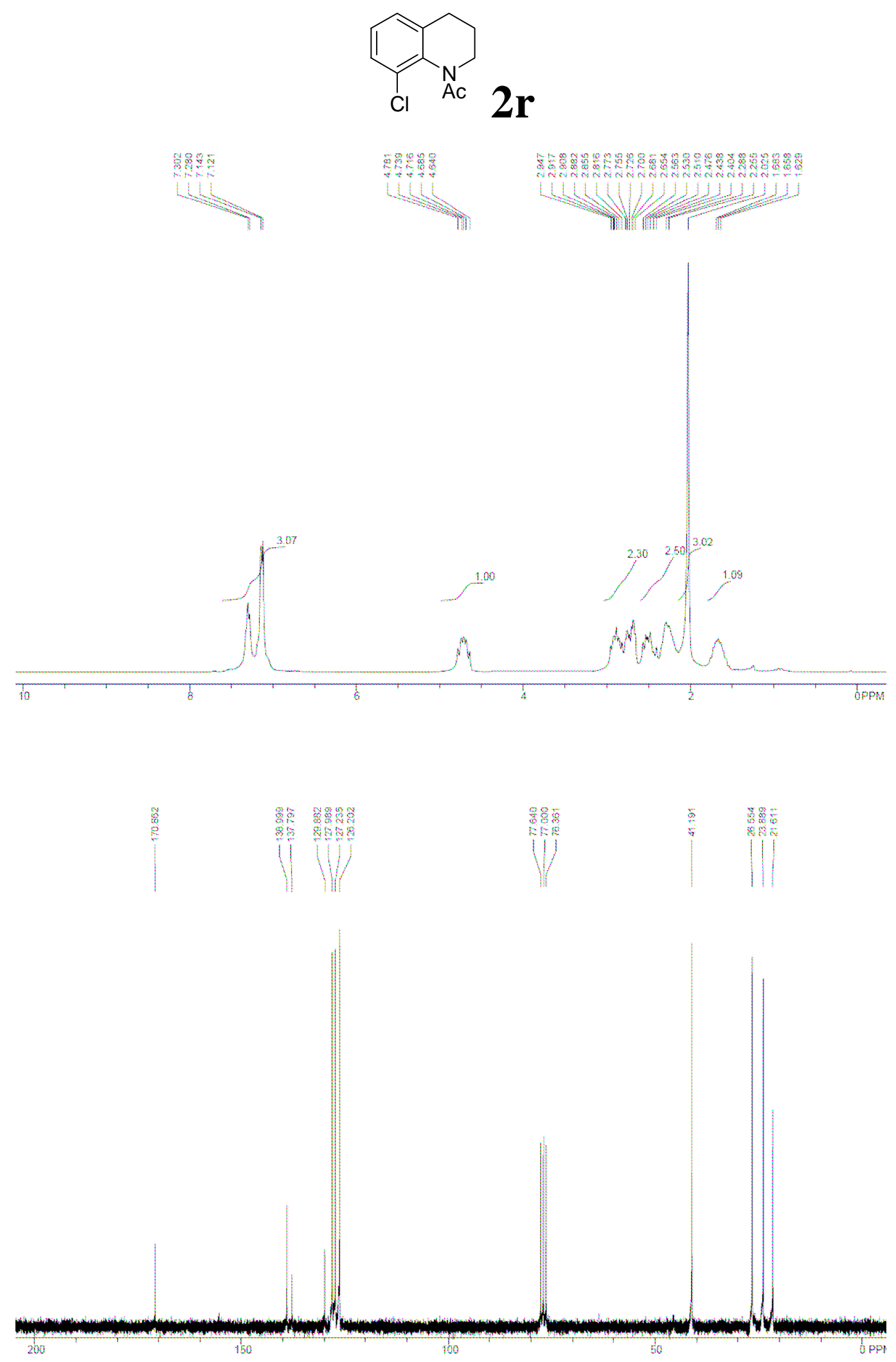


\section{References:}

(1) Mossi, Waldo; Klaus, Alfred J.; Rys, Paul; Helv. Chim. Acta; 1992; 2531-2537.

(2) Wang, Jin-Xian; Xing, Zhiliang; Zhang, Manli; Hu, Yulai; J. Chem. Res. Miniprint; 1998; 886-896.

(3) Mulhern, Thomas A.; Davis, Mark; Krikke, James J.; Thomas, James A.; J. Org. Chem.; 1993; 5537-5540.

(4) Kikugawa, Yasuo; Mitsui, Kimiyo; Chem. Lett.; 1993; 1369-1372.

(5) El-Sheikh, Mustafa I.; Marks, Alan; Biehl, Edward R.; J. Org. Chem.; 1981; 3256-3259.

(6) Kasahara, Akira; Izumi, Taeko; Murakami, Satoshi; Miyamoto, Kazuhiro; Hino, Toshimi; J. Heterocycl. Chem.; 1989; 1405-1413.

(7) Shepard; Shonle; J. Am. Chem. Soc.; 1948; 1979.

(8) Abela Medici,A.J. et al.; J. Chem. Soc. Perkin Trans. 1; 1977; 2517-2520.

(9) Rae; Aust. J. Chem.; 1974; 2621-2623.

(10) Rosowsky, Andre; Mota, Clara E.; Queener, Sherry F.; J. Heterocycl. Chem.; 1996; 1959-1966.

(11) Renneberg, Dorte; Dervan, Peter B.; J. Am. Chem. Soc.; 2003; 125, 5707 - 5716.

(12) Imbs, J.; Leibenguth, A.; Koffel, J. C.; Jung, L.; Arzneim. Forsch.; 1982; 32; 503-508.

(13) Kato et al.; Chem. Pharm. Bull.; 1971; 19; 832. 


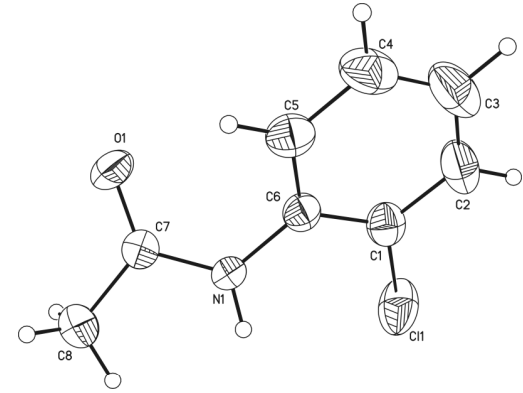

2a

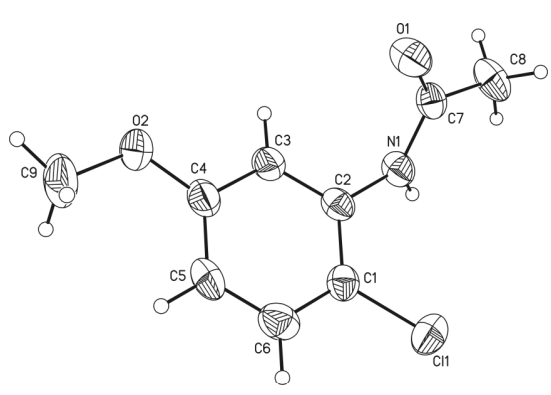

$2 m$

Table S1. Crystallographic data and refinement details for compounds $\mathbf{2 a}, \mathbf{2 m}$

\begin{tabular}{|c|c|c|}
\hline & $\mathbf{2 a}$ & $2 \mathrm{~m}$ \\
\hline Formula & $\mathrm{C}_{8} \mathrm{H}_{8} \mathrm{ClNO}$ & $\mathrm{C}_{9} \mathrm{H}_{9} \mathrm{ClNO}_{2}$ \\
\hline M/g mol-1 & 169.60 & 198.62 \\
\hline Color & colourless & colourless \\
\hline Crystal size/mm & $0.40 \times 0.12 \times 0.12 \mathrm{~mm}$ & $0.50 \times 0.15 \times 0.15 \mathrm{~mm}$ \\
\hline Crystal system, & Orthorhombic & Monoclinic \\
\hline Space group & Pbca & $\mathrm{P} 2(1) / \mathrm{c}$ \\
\hline $\mathrm{a} / \AA$ & $10.516(2)$ & $9.3602(19)$ \\
\hline $\mathrm{b} / \AA$ & $9.5540(19)$ & $12.290(3)$ \\
\hline c/ $\AA$ & $16.670(3)$ & $8.5900(17)$ \\
\hline$\alpha /^{\circ}$ & 90 & 90.00 \\
\hline$\beta /{ }^{\circ}$ & 90 & $105.34(3)$ \\
\hline$\gamma /{ }^{\circ}$ & 90 & 90.00 \\
\hline $\mathrm{V} / \AA^{3}$ & $1674.9(6)$ & $953.0(3)$ \\
\hline$\rho$ calc $/ \mathrm{g} \mathrm{cm}^{-3}$ & 1.345 & 1.384 \\
\hline $\mathrm{Z}$ & 8 & 4 \\
\hline $\mathrm{F}(000)$ & 704 & 412 \\
\hline Radiation used & Mo-K $\alpha$ & Mo-K $\alpha$ \\
\hline
\end{tabular}




\begin{tabular}{|c|c|c|}
\hline$\mu / \mathrm{mm}^{-1}$ & 0.395 & 0.366 \\
\hline $\mathrm{T} / \mathrm{K}$ & 293(2) K & 293(2) K \\
\hline hkl range & $\begin{array}{c}-12<=\mathrm{h}<=12,-11<=\mathrm{k}<=11, \\
-19<=\mathrm{l}<=19\end{array}$ & $\begin{array}{c}-11<=\mathrm{h}<=11,-15<=\mathrm{k}<=15, \\
-10<=1<=10\end{array}$ \\
\hline$\theta$ range $/^{\circ}$ & $2.44-25.01$ & $2.26-26.49$ \\
\hline Reflections measured & 5828 & 4131 \\
\hline Unique reflections $\left(\mathrm{R}_{\text {int }}\right)$ & $1465(0.0337)$ & $1840(0.0603)$ \\
\hline Reflections, $\mathrm{n}[\mathrm{I} \geq 2 \sigma(\mathrm{I})]$ & 776 & 984 \\
\hline Refinement method & $\begin{array}{l}\text { Full-matrix least-squares } \\
\text { on } \mathrm{F}^{\wedge} 2\end{array}$ & $\begin{array}{l}\text { Full-matrix least-squares } \\
\text { on } \mathrm{F}^{\wedge} 2\end{array}$ \\
\hline $\begin{array}{l}\text { Refined parameters/restraints } \\
\text { R1 }[\mathrm{I}>2 \sigma]\end{array}$ & $105 / 0$ & $123 / 0$ \\
\hline R1 (all data) & $0.0323, w R 2=0.0634$ & $0.0551, w \mathrm{R} 2=0.1391$ \\
\hline Goodness-of-fit on F2 & $0.0670, w R 2=0.0663$ & $0.0977, w R 2=0.1548$ \\
\hline Largest diff. peak and hole/e. $\AA$ & 0.936 & 1.004 \\
\hline-3 & 0.212 and -0.153 & 0.263 and -0.280 \\
\hline
\end{tabular}

Table S2. Selected bond distances $(\AA)$ and angles $\left(^{\circ}\right)$ for compounds $\mathbf{2 a}, \mathbf{2 m}$

\section{Compound 2a}

$\begin{array}{cc}\mathrm{Cl}(1)-\mathrm{C}(1) & 1.740(2) \\ \mathrm{O}(1)-\mathrm{C}(7) & 1.229(3) \\ \mathrm{N}(1)-\mathrm{C}(7) & 1.345(3) \\ \mathrm{N}(1)-\mathrm{C}(6) & 1.409(3) \\ \mathrm{N}(1)-\mathrm{HN} & 0.852(18) \\ \mathrm{C}(1)-\mathrm{C}(2) & 1.374(3) \\ \mathrm{C}(1)-\mathrm{C}(6) & 1.387(3) \\ \mathrm{C}(2)-\mathrm{C}(3) & 1.370(3)\end{array}$




\begin{tabular}{|c|c|}
\hline $\mathrm{C}(2)-\mathrm{H}(2)$ & 0.9300 \\
\hline $\mathrm{C}(3)-\mathrm{C}(4)$ & $1.379(3)$ \\
\hline $\mathrm{C}(3)-\mathrm{H}(3)$ & 0.9300 \\
\hline $\mathrm{C}(4)-\mathrm{C}(5)$ & $1.379(3)$ \\
\hline $\mathrm{C}(4)-\mathrm{H}(4)$ & 0.9300 \\
\hline$C(5)-C(6)$ & $1.390(3)$ \\
\hline $\mathrm{C}(5)-\mathrm{H}(5)$ & 0.9300 \\
\hline $\mathrm{C}(7)-\mathrm{C}(8)$ & $1.500(3)$ \\
\hline $\mathrm{C}(8)-\mathrm{H}(8 \mathrm{~A})$ & 0.9600 \\
\hline $\mathrm{C}(8)-\mathrm{H}(8 \mathrm{~B})$ & 0.9600 \\
\hline $\mathrm{C}(8)-\mathrm{H}(8 \mathrm{C})$ & 0.9600 \\
\hline $\mathrm{C}(7)-\mathrm{N}(1)-\mathrm{C}(6)$ & $125.3(2)$ \\
\hline $\mathrm{C}(7)-\mathrm{N}(1)-\mathrm{HN}$ & 118.1(14) \\
\hline $\mathrm{C}(6)-\mathrm{N}(1)-\mathrm{HN}$ & $116.5(14)$ \\
\hline$C(2)-C(1)-C(6)$ & $121.0(2)$ \\
\hline $\mathrm{C}(2)-\mathrm{C}(1)-\mathrm{Cl}(1)$ & $118.2(2)$ \\
\hline $\mathrm{C}(6)-\mathrm{C}(1)-\mathrm{Cl}(1)$ & $120.81(17)$ \\
\hline $\mathrm{C}(3)-\mathrm{C}(2)-\mathrm{C}(1)$ & 119.7(3) \\
\hline $\mathrm{C}(3)-\mathrm{C}(2)-\mathrm{H}(2)$ & 120.1 \\
\hline $\mathrm{C}(1)-\mathrm{C}(2)-\mathrm{H}(2)$ & 120.1 \\
\hline $\mathrm{C}(2)-\mathrm{C}(3)-\mathrm{C}(4)$ & $120.6(3)$ \\
\hline $\mathrm{C}(2)-\mathrm{C}(3)-\mathrm{H}(3)$ & 119.7 \\
\hline $\mathrm{C}(4)-\mathrm{C}(3)-\mathrm{H}(3)$ & 119.7 \\
\hline $\mathrm{C}(5)-\mathrm{C}(4)-\mathrm{C}(3)$ & $119.6(3)$ \\
\hline $\mathrm{C}(5)-\mathrm{C}(4)-\mathrm{H}(4)$ & 120.2 \\
\hline $\mathrm{C}(3)-\mathrm{C}(4)-\mathrm{H}(4)$ & 120.2 \\
\hline$C(4)-C(5)-C(6)$ & $120.6(2)$ \\
\hline $\mathrm{C}(4)-\mathrm{C}(5)-\mathrm{H}(5)$ & 119.7 \\
\hline
\end{tabular}




\begin{tabular}{lc}
\hline $\mathrm{C}(6)-\mathrm{C}(5)-\mathrm{H}(5)$ & 119.7 \\
$\mathrm{C}(1)-\mathrm{C}(6)-\mathrm{C}(5)$ & $118.5(2)$ \\
$\mathrm{C}(1)-\mathrm{C}(6)-\mathrm{N}(1)$ & $119.8(2)$ \\
$\mathrm{C}(5)-\mathrm{C}(6)-\mathrm{N}(1)$ & $121.7(2)$ \\
$\mathrm{O}(1)-\mathrm{C}(7)-\mathrm{N}(1)$ & $123.4(2)$ \\
$\mathrm{O}(1)-\mathrm{C}(7)-\mathrm{C}(8)$ & $121.5(2)$ \\
$\mathrm{N}(1)-\mathrm{C}(7)-\mathrm{C}(8)$ & $115.1(2)$ \\
$\mathrm{C}(7)-\mathrm{C}(8)-\mathrm{H}(8 \mathrm{~A})$ & 109.5 \\
$\mathrm{C}(7)-\mathrm{C}(8)-\mathrm{H}(8 \mathrm{~B})$ & 109.5 \\
$\mathrm{H}(8 \mathrm{~A})-\mathrm{C}(8)-\mathrm{H}(8 \mathrm{~B})$ & 109.5 \\
$\mathrm{C}(7)-\mathrm{C}(8)-\mathrm{H}(8 \mathrm{C})$ & 109.5 \\
$\mathrm{H}(8 \mathrm{~A})-\mathrm{C}(8)-\mathrm{H}(8 \mathrm{C})$ & 109.5 \\
$\mathrm{H}(8 \mathrm{~B})-\mathrm{C}(8)-\mathrm{H}(8 \mathrm{C})$ & 109.5
\end{tabular}

Compound 2m

C(11)-C(1)

1.739(3)

$\mathrm{O}(1)-\mathrm{C}(7)$

1.224(3)

$\mathrm{O}(2)-\mathrm{C}(4)$

1.367(4)

$\mathrm{O}(2)-\mathrm{C}(9)$

1.420(4)

$\mathrm{N}(1)-\mathrm{C}(7)$

1.347(4)

$\mathrm{N}(1)-\mathrm{C}(2)$

1.408(4)

$\mathrm{N}(1)-\mathrm{HN}(1)$

0.79(3)

$\mathrm{C}(1)-\mathrm{C}(6)$

1.378(5)

$\mathrm{C}(1)-\mathrm{C}(2)$

1.391(4)

$\mathrm{C}(2)-\mathrm{C}(3)$

$1.388(4)$

C(3)-C(4)

1.376(4)

$\mathrm{C}(3)-\mathrm{H}(3)$

0.9300

C(4)-C(5)

1.383(4)

$\mathrm{C}(5)-\mathrm{C}(6)$

1.373(5) 


\begin{tabular}{|c|c|}
\hline $\mathrm{C}(5)-\mathrm{H}(5)$ & 0.9300 \\
\hline $\mathrm{C}(6)-\mathrm{H}(6)$ & 0.9300 \\
\hline $\mathrm{C}(7)-\mathrm{C}(8)$ & $1.498(4)$ \\
\hline $\mathrm{C}(8)-\mathrm{H}(8 \mathrm{~A})$ & 0.9600 \\
\hline $\mathrm{C}(8)-\mathrm{H}(8 \mathrm{~B})$ & 0.9600 \\
\hline $\mathrm{C}(8)-\mathrm{H}(8 \mathrm{C})$ & 0.9600 \\
\hline $\mathrm{C}(9)-\mathrm{H}(9 \mathrm{~A})$ & 0.9600 \\
\hline $\mathrm{C}(9)-\mathrm{H}(9 \mathrm{~B})$ & 0.9600 \\
\hline $\mathrm{C}(9)-\mathrm{H}(9 \mathrm{C})$ & 0.9600 \\
\hline $\mathrm{C}(4)-\mathrm{O}(2)-\mathrm{C}(9)$ & $118.6(3)$ \\
\hline $\mathrm{C}(7)-\mathrm{N}(1)-\mathrm{C} 2$ & $124.8(3)$ \\
\hline $\mathrm{C}(7)-\mathrm{N}(1)-\mathrm{H}(\mathrm{N} 1)$ & $122(2)$ \\
\hline $\mathrm{C}(2)-\mathrm{N}(1)-\mathrm{H}(\mathrm{N} 1)$ & $113(2)$ \\
\hline $\mathrm{C}(6)-\mathrm{C}(1)-\mathrm{C}(2)$ & $120.6(3)$ \\
\hline $\mathrm{C}(6)-\mathrm{C}(1)-\mathrm{C}(11)$ & $120.0(2)$ \\
\hline $\mathrm{C}(2)-\mathrm{C}(1)-\mathrm{C}(11)$ & $119.3(2)$ \\
\hline $\mathrm{C}(3)-\mathrm{C}(2)-\mathrm{C}(1)$ & $118.0(3)$ \\
\hline $\mathrm{C}(3)-\mathrm{C}(2)-\mathrm{N}(1)$ & $120.7(3)$ \\
\hline $\mathrm{C}(1)-\mathrm{C}(2)-\mathrm{N}(1)$ & $121.3(3)$ \\
\hline $\mathrm{C}(4)-\mathrm{C}(3)-\mathrm{C}(2)$ & $121.4(3)$ \\
\hline $\mathrm{C}(4)-\mathrm{C}(3)-\mathrm{H}(3)$ & 119.3 \\
\hline $\mathrm{C}(2)-\mathrm{C}(3)-\mathrm{H}(3)$ & 119.3 \\
\hline $\mathrm{O}(2)-\mathrm{C}(4)-\mathrm{C}(3)$ & $115.0(3)$ \\
\hline $\mathrm{O}(2)-\mathrm{C}(4)-\mathrm{C}(5)$ & $125.3(3)$ \\
\hline $\mathrm{C}(3)-\mathrm{C}(4)-\mathrm{C}(5)$ & 119.7(3) \\
\hline $\mathrm{C}(6)-\mathrm{C}(5)-\mathrm{C}(4)$ & $119.7(3)$ \\
\hline $\mathrm{C}(6)-\mathrm{C}(5)-\mathrm{H}(5)$ & 120.1 \\
\hline $\mathrm{C}(4)-\mathrm{C}(5)-\mathrm{H}(5)$ & 120.1 \\
\hline
\end{tabular}




\begin{tabular}{ll}
\hline $\mathrm{C}(5)-\mathrm{C}(6)-\mathrm{C}(1)$ & $120.5(3)$ \\
$\mathrm{C}(5)-\mathrm{C}(6)-\mathrm{H}(6)$ & 119.7 \\
$\mathrm{C}(1)-\mathrm{C}(6)-\mathrm{H}(6)$ & 119.7 \\
$\mathrm{O}(1)-\mathrm{C}(7)-\mathrm{N}(1)$ & $122.0(3)$ \\
$\mathrm{O}(1)-\mathrm{C}(7)-\mathrm{C}(8)$ & $122.9(3)$ \\
$\mathrm{N}(1)-\mathrm{C}(7)-\mathrm{C}(8)$ & $115.1(3)$ \\
$\mathrm{C}(7)-\mathrm{C}(8)-\mathrm{H}(8 \mathrm{~A})$ & 109.5 \\
$\mathrm{C}(7)-\mathrm{C}(8)-\mathrm{H}(8 \mathrm{~B})$ & 109.5 \\
$\mathrm{H}(8 \mathrm{~A})-\mathrm{C}(8)-\mathrm{H}(8 \mathrm{~B})$ & 109.5 \\
$\mathrm{C}(7)-\mathrm{C}(8)-\mathrm{H}(8 \mathrm{C})$ & 109.5 \\
$\mathrm{H}(8 \mathrm{~A})-\mathrm{C}(8)-\mathrm{H}(8 \mathrm{C})$ & 109.5 \\
$\mathrm{H}(8 \mathrm{~B})-\mathrm{C}(8)-\mathrm{H}(8 \mathrm{C})$ & 109.5 \\
$\mathrm{O}(2)-\mathrm{C}(9)-\mathrm{H}(9 \mathrm{~A})$ & 109.5 \\
$\mathrm{O}(2)-\mathrm{C}(9)-\mathrm{H}(9 \mathrm{~B})$ & 109.5 \\
$\mathrm{H}(9 \mathrm{~A})-\mathrm{C}(9)-\mathrm{H}(9 \mathrm{~B})$ & 109.5 \\
$\mathrm{O}(2)-\mathrm{C}(9)-\mathrm{H}(9 \mathrm{C})$ & 109.5 \\
$\mathrm{H}(9 \mathrm{~A})-\mathrm{C}(9)-\mathrm{H}(9 \mathrm{C})$ & 109.5 \\
$\mathrm{H}(9 \mathrm{~B})-\mathrm{C}(9)-\mathrm{H}(9 \mathrm{C})$ & 109.5 \\
& \\
\hline
\end{tabular}

